\title{
Immunohistochemical basigin expression level in thyroid cancer tissues
}

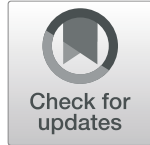

\author{
Wan-Ping Guo ${ }^{1 \dagger}$, Deng Tang ${ }^{1 \dagger}$, Yu-Yan Pang ${ }^{1}$, Xiao-Jiao Li², Gang Chen ${ }^{1}$, Zhi-Guang Huang ${ }^{1}$, Xiao-Zhun Tang ${ }^{3}$, \\ Qin-Qiao Lai ${ }^{1}$, Jin-Yan Gan ${ }^{1}$, Xiao-Li Huang ${ }^{1}$, Xiao-Fan Liu' ${ }^{1}$, Zhi-Xiao Wei ${ }^{2^{*}}$ and Wei Ma ${ }^{1 *}$ (D)
}

\begin{abstract}
Background: Thyroid cancer (TC) is the most common endocrine malignancy; basigin (also known as BSG) plays a crucial role in tumor cell invasion, metastasis, and angiogenesis. This study was designed to identify the change of BSG expression in TC and its possible potential mechanism.

Methods: The BSG expression levels in TC were demonstrated using data collected from in-house immunohistochemical (IHC), RNA-sequencing (RNA-seq), microarrays, and literatures. Integrated analysis was performed to determined BSG expression levels in TC comprehensively. The Gene Ontology (GO) and Kyoto Encyclopedia of Genes and Genomes (KEGG) enrichment analyses were performed with the integration of BSG co-expressed genes and differentially expressed genes (DEGs) in TC tissues to explore the potential mechanisms of BSG in TC.

Results: The protein expression level of BSG was significantly higher in TC cases based on the IHC experiments. In addition, the combined SMD for BSG expression was $0.39(p<0.0001)$, the diagnostic odds ratio was 3.69, and the AUC of the sROC curve was 0.6986 using 1182 TC cases and 437 non-cancerous cases from 17 independent datasets.

Furthermore, BSG co-expressed genes tended to be enriched in gene terms of the extracellular matrix (ECM), cell adhesion, and cell-cell interactions. The expression levels of nine hub BSG co-expressed genes were markedly upregulated in TC cases.
\end{abstract}

Conclusion: BSG expression levels were closely correlated with the progression of TC and may affect the signals of the $\mathrm{ECM}$, cell adhesion, and cell-cell interactions.

Keywords: Basigin, Thyroid cancer, Thyroid papillary carcinoma, Tissue microarray, RNA-sequencing

\section{Background}

Thyroid cancer (TC) is the most common endocrine malignancy, and its prevalence has increased dramatically in the last few years [1-3]. Histological types of TC include papillary carcinoma (PTC), follicular carcinoma

\footnotetext{
* Correspondence: weizhixiao196493@126.com; mawei_gxmu@163.com

+Wan-Ping Guo and Deng Tang contributed equally to this work.

${ }^{2}$ Department of Nuclear Medicine, First Affiliated Hospital of Guangxi Medical

University, 6 Shuangyong Road, Nanning 530021, Guangxi Zhuang

Autonomous Region, People's Republic of China

${ }^{1}$ Department of Pathology, First Affiliated Hospital of Guangxi Medical

University, 6 Shuangyong Road, Nanning 530021, Guangxi Zhuang

Autonomous Region, People's Republic of China

Full list of author information is available at the end of the article
}

(FTC), anaplastic carcinoma (ATC)/undifferentiated thyroid carcinoma(UTC), and medullary carcinoma (MTC) [4], and there have been different mutational profiles in these subtypes. Recent studies have confirmed a few molecular markers that have allowed better understanding of the molecular mechanisms of TC, for instance, BRAF and RAS point mutations, RET/PTC and PAX8/PPAR gene rearrangements, etc [5-7]. In addition, the latest study found that Substance P/neurokinin-1 receptor (SP/NK-1R) system was over-expressed in TC than that in normal thyroid tissues via immunohistochemical study, which could promote the migration and invasion of cancer cells [8]. Targeting certain molecules 
associated with initiation and progression of TC will be a significant and promising research area. Therefore, it was critical to continue to study the molecular mechanisms involved in $\mathrm{TC}$, which may provide new strategies for the diagnosis and treatment of $\mathrm{TC}$ patients in the future.

Basigin (also known as BSG, CD147, or Extracellular Matrix Metalloproteinase inducer [EMMPRIN]) is a single pass type 1 transmembrane protein that plays a crucial part in developmental processes, wound healing, nutrient transport, inflammation, arthritis, and microbial pathologies. BSG has also been verified as a potential stimulator of matrix metalloproteinases (MMPs) and is considered to be a prognostic marker in cancer [9]. Accumulating studies have found that BSG was overexpressed in many different human tumor cell types, such as those caused by brain cancer, colon cancer, cervical cancer, and endometrial cancer. It also played a crucial role in tumor cell invasion, metastasis, and angiogenesis [10-14]. The role of BSG in cancer suggests that it could be an effective therapeutic target. For instance, exciting clinical progress has been made in hepatocellular carcinoma (HCC) treatment by means of BSGdirected monoclonal antibodies [15].

As for TC, BSG also played an important role in the tumorigenicity, invasion, metastasis, and degree of dedifferentiation [16, 17]. Recently, researchers have identified that BSG expression regulates tumor cell glycolysis, resulting in the progression of TC $[17,18]$. In addition, researchers have also found that BSG gene silencing leads to growth inhibition of thyroid medullary carcinoma TT cells and alteration of the cell cycle [17]. Moreover, it was proved that BSG was involved in the invasiveness of FTC cells via regulation of MMPs [19]. For differentiated carcinoma (DTC), the expression of BSG and MMP-2 may be an important feature, and the expression of BSG may be useful to predict the prognosis of DTC patients [20, 21]. Furthermore, a study demonstrated that BSG inhibition may be a therapeutic target for TC patients [18].

In summary, accumulating evidence suggested that BSG exerts a variety of functions in TC progression. However, until now, only one study mentioned a possible molecular mechanism underlying TC: miR-125a-5p regulated BSG and was negatively correlated with its expression and function [18]. Within this context, little was known about the molecular mechanism of BSG in $\mathrm{TC}$, and it was essential to further investigate its expression and function.

In this study, we analyzed cases gathered from immunohistochemistry (IHC), RNA-sequencing (RNA-seq), and gene microarray data to provide evidence to attest to the clinical value of BSG in TC. Co-expressed and differentially expressed genes (DEGs) of BSG in TC, along with the corresponding intersection genes and hub genes, were also obtained. In addition, Gene Ontology (GO) and Kyoto Encyclopedia of Genes and Genomes (KEGG) enrichment analyses were performed to intersect genes and explore the potential mechanisms of BSG in TC. We hope that our findings will broaden the horizon for future studies of TC and BSG.

\section{Materials and methods \\ BSG expression level in TC \\ Detecting protein expression levels of BSG by in-house immunohistochemistry}

Two tissue microarrays (THC961 and THC1021) which contained 23 non-cancerous thyroid tissues and 125 TC (including PTC, FTC, MTC, and ATC) tissues were obtained from Fanpu Biotech, Inc. (Guilin, China). In addition, a number of 64 non-cancerous thyroid tissues and 46 TC tissues were collected from the Department of Pathology, First Affiliated Hospital of Guangxi Medical University, from March 1 to December 1, 2018. The study was approved by the Ethical Committee of the First Affiliated Hospital of Guangxi Medical University. And all patients provided written informed consents for use of their samples in study. Subsequently, pathologists (Wei-jia Mo and Gang Chen) independently evaluated all slides using a semi-quantitative scoring system without knowing the clinical results in advance to classify the staining intensity and the percentage of positive cancer cells. The staining intensity score was divided into 0 (negative), 1 (weak), 2 (medium), or 3 (strong). And the proportion of staining score set as $0(<10 \%), 1$ (11$25 \%), 2$ (26-50\%), $3(51-75 \%)$, or 4 (76-100\%). Finally, the final immune response score was determined by combining the intensity and proportion scores. In addition, a correlation analysis was constructed to determine the relationships between BSG expression levels, clinicopathological characteristics, and patient prognosis. Furthermore, we supplemented the immunohistochemical results of the BSG expression levels in thyroid cancer by searching the available literature and The Human Protein Atlas [22].

\section{BSG mRNA expression in TC samples from TCGA RNA-seq data}

Using The Cancer Genome Atlas (TCGA) database, we downloaded BSG expression data related to TC and para-cancerous tissues [23]. Then, the downloaded data were integrated and transferred into the quantile normalized $\log 2$ format to improve measurement accuracy. SPSS 25.0 (SPSS Inc. Chicago, IL, USA) was used to calculate the mean BSG expression and standard deviation (SD) in 505 TC tissues and 59 non-cancer tissues, incorporating clinical information gathered from the TCGA database. Furthermore, a Kaplan-Meier survival 
curve combined with TC patients' survival data was constructed using GraphPad Prism Version 7.0, in order to estimate the prognostic capacity of BSG in TC.

\section{BSG expression in TC tissues from other microarray data}

This study included The Gene Expression Database (GEO) [24], ArrayExpress [25], Oncomine [26], and The Sequence Read Archive (SRA) [27] in order to search both Chinese and English medical literature for microarray data of BSG to analyze its clinical value in TC. Our search included data entered into the databases from the beginning to February 21, 2020. The retrieval formula of this study was as follows: (cancer OR carcinoma OR adenocarcinoma OR tumour OR tumor OR malignanc* OR neoplas*) AND (Thyroid OR Thyroidea OR Glandula thyroidea) AND (mRNA OR gene OR "messenger RNA"). Finally, only microchip data relevant to human samples that included BSG expression in TC tissues and non-cancerous tissues were selected.

\section{Searching the literature for BSG expression}

In order to supplement the new data on BSG expression in recent studies, we conducted a comprehensive literature search of electronic databases (including PubMed, Embase, Web of Science, Wiley Online Library, SpringerLink, Chinese National Knowledge Infrastructure, Chinese Biomedical Literature Database, Chinese VIP, and Wanfang database) to obtain eligible studies. The search retrieval formula for these databases was as follows: (cancer OR carcinoma OR adenocarcinoma OR tumour OR tumor OR malignanc* OR neoplas*) AND (Thyroid OR Thyroidea OR Glandula thyroidea) AND ("Basigin" OR "Ok blood group" OR "CD147" OR "EMMPRIN"). Only studies that included human BSG expression levels were selected.

\section{Comprehensive analysis of BSG expression in TC}

Data from four types of sources (IHC, RNA-seq, microarrays, and literature) were combined using Stata Version 12.0 in order to perform a comprehensive analysis to determine BSG expression levels in TC, and the standard mean difference (SMD) and 95\% confidence interval (CI) were calculated. Furthermore, the summary receiver operating characteristic (sROC) curve was used to distinguish TC tissues from non-cancerous tissues, which were gathered from the above databases. Sensitivity and specificity, as well as the corresponding likelihood ratio (LR) and diagnostic odds ratio (DOR) were calculated using meta-disk Version 1.4 [28-30].

\section{Validation of the expression of BSG using cell line data from CCLE}

A search for the term "BSG" was performed on the Cancer Cell Line Encyclopedia (CCLE) database [31], and a heat-map based on the expression of BSG in different TC cell lines was constructed using HemI (Heatmap Illustrator, version 1.0).

\section{The potential mechanism of BSG expression in thyroid cancer Identification of BSG co-expressed genes and differentially expressed genes (DEGs) in TC}

To obtain the hub genes of BSG in TC, we first searched the cBioPortal for Cancer Genomics [32] and found the co-expressed genes of BSG. The DEGs from all gene microarray datasets and RNA-seq data were obtained using the RRA method. This method uses a probabilistic model for aggregation, which is resilient to noise, and can calculate the probability of significance of all elements and perform the final ranking [33-37]. The cutoff $P$ value for the RRA was set as 0.05 .

Eventually, the genes that intersected with the coexpressed genes and DEGs were selected for subsequent work.

\section{GO and KEGG clustering analyses of BSG co-expressed genes and DEGs in TC}

The BSG co-expressed genes and DEGs in TC were uploaded into a function annotation portal via The Database for Annotation, Visualization, and Integrated Discovery (DAVID) [38], an R studio software that provides a systematic and comprehensive analysis for gene lists.

\section{Identification of BSG hub genes in TC}

The protein interactions of BSG co-expressed genes and DEGs were obtained using the Search Tool for the Retrieval of Interacting Genes (STRING) network database, and we set the minimum required interaction score at 0.7. We then used CytoHubba, a plug-in of Cytoscape, which can identify hub objects and sub-networks from complex interactome to calculate gene nodes present in the interaction network. We imported PPI results of STRING website into Cytoscape, selected the CytoHubba plug-in, and calculated the top ten genes through MCC algorithm which has a better performance on the precision of predicting essential proteins from the yeast PPI network [39]. And the network interface will show the connection of these hub nodes in the network. The darker the node color, the higher the score. Ultimately, the top 10 genes were selected as BSG hub genes in TC.

\section{Statistical analysis}

SPSS 25.0 software was chosen for data analysis, which included an independent Student's $t$ test to determine the significance of BSG expression in two groups and a univariate analysis for three or more groups. A $P$ value below 0.05 was considered statistically significant. Scatter diagrams whose data ranged between normal and TC 
tissues were prepared by the GraphPad Prism 7.0 software. In addition, we established a ROC curve to evaluate the diagnostic role of BSG in TC and differentiated $\mathrm{TC}$ and non-cancerous tissue by area under the curve (AUC), which can be used as a diagnostic. Moreover, according to the AUC, the diagnostic efficacy can be divided into three levels: low (0.5-0.7), medium (0.7-0.9), and high (0.9-1.0).

\section{Results}

\section{Immunohistochemical investigation for BSG protein} expression levels

This study included 171 cases of TC tissues and 87 cases of non-cancerous tissues, which were collected from the First Affiliated Hospital of Guangxi Medical University (Fig. 1a-h). Based on these samples, the BSG protein expression in the 171 cases of TC tissues was notably higher than that in the 87 cases of non-cancerous tissues $(7.6 \pm 1.366$ vs. $1.32 \pm 1.749, p<0.0001)$ (Fig. 1i). The AUC of BSG protein expression used to distinguish between TC cases and non-cancerous cases was 0.985 (95\%CI 0.971 to $0.999, p<0.0001$ ). The sensitivity and specificity were 0.912 and 0.966 , respectively (Fig. 1j). In addition, the relationship between BSG expression and clinical parameters was shown supplementary Table S1.

\section{BSG mRNA expression in TC samples}

In total, we collected $505 \mathrm{TC}$ samples and 59 noncancerous samples for RNA-seq data analysis. Corresponding clinical information was also collected and was shown in Table 1. The BSG mRNA expression level in the TC samples was dramatically higher than that in the non-cancerous samples (3.8 \pm 0.056 vs. $3.76 \pm 0.042, p<$ 0.0001). Furthermore, the AUC of BSG mRNA expression was $0.7363(95 \% \mathrm{CI} 0.6743$ to $0.7983, p<0.0001)$, and the sensitivity and specificity were 0.602 and 0.814 , respectively (Fig. 2a, b). Subsequently, the prognostic value of BSG expression was inspected. The KaplanMeier survival curve analysis revealed that TC patients with low BSG expression levels were more likely to have worse overall survival rates (Fig. 2c, d) than those with high BSG expression levels (log-rank $p=0.5027$ ). In addition, the HR value was 0.6988 (95\% CI 0.2564 to 1.905). More interestingly, TC patients with high BSG expression levels tended to have worse rates of diseasefree survival than those with low BSG expression levels (log-rank $p=0.0947)$, and the HR value was 10.98 (95\%CI 0.6606 to 182.5 ).

\section{BSG expression in TC tissues according to the microarray data analysis}

After the retrieval using the GEO database, 15 microarrays of BSG expression datasets were selected for our study. These microarrays contained 506 TC cases and
291 non-cancerous cases (Fig. 3, Table 2). Of these 15 microarrays, 9 (GSE27155, GSE53072, GSE6004, GSE58545, GSE35570, GSE53157, GSE50901, GSE60542, and GSE58689) showed that the expression levels of BSG in TC cases were slightly higher than that in noncancerous cases. In order to further analyze the BSG expression levels in these microarrays, we merged the microarray data and performed a meta-analysis. The combined SMD for BSG was 0.11 (95\%CI - 0.04 to 0.25$)$ based on the fixed effect model (Supplementary Figure S1A). The $I$ squared value and the $P$ value were $0.0 \%$ and 0.570 , respectively. A sensitivity analysis of BSG expression levels in TC tissues and non-cancerous tissues was performed to show the differences among the microarrays (Supplementary Figure S1B). In addition, the publication bias was assessed by means of a funnel plot (Supplementary Figures S1C and S1D). The publication bias was not significant for this meta-analysis of BSG expression according to Begg's test $(p=0.805)$ and Egger's test $(p=0.745)$. Furthermore, the funnel plot also displayed a symmetric shape.

The combined sensitivity of BSG was 0.47 (95\%CI 0.42 to $0.51, p<0.0001)$, and the specificity was $0.74(95 \% \mathrm{CI}$ 0.69 to $0.79, p<0.0001$ ) with a positive likelihood ratio of 1.53 (95\%CI 1.26 to $1.86, p=0.9913$ ), a negative likelihood ratio of 0.79 ( $95 \% \mathrm{CI} 0.69$ to $0.90, p=0.0367$ ), and a diagnostic odds ratio of 2.38 (95\%CI 1.65 to $3.43, p=$ 0.9277). Most importantly, the AUC of BSG reached 0.6539 , showing that BSG has a good capacity to differentiate TC tissues from the non-cancerous tissues (Supplementary Figure S2).

\section{Integrated analysis of the in-house}

immunohistochemistry, RNA-seq, microarray, and BSG expression data reported in the literature

In order to comprehensively and systematically analyze the BSG expression level in TC, we performed a metaanalysis combining all sources of data, which included 1182 TC cases and 437 non-cancerous cases $(n=17)$. The combined SMD for BSG expression was 0.39 (95\%CI -0.19 to 0.97$)$ according to a random effect model ( $I$-squared value, 94.7\%). In addition, the $P$ value was $<0.0001$, confirming a significant difference in the sensitivity analysis (Fig. 4a, b). Moreover, the publication bias was not significant according to Begg's test $(p=$ 0.323 ) and Egger's test ( $p=0.508)$ (Fig. 4c, d). The combined sensitivity of BSG was 0.59 (95\%CI 0.56 to $0.62, p$ $<0.0001)$, and the specificity was 0.80 (95\%CI 0.75 to $0.83, p<0.0001)$ with a positive likelihood ratio of 1.89 (95\%CI 1.30 to $2.74, p<0.0001$ ), a negative likelihood ratio of 0.59 (95\% CI 0.45 to $0.79, p<0.0001$ ), and a diagnostic odds ratio of 3.69 (95\% CI 1.99 to $6.87, p<$ 0.0001). Ultimately, the AUC of the sROC curve, which estimated the capacity of BSG expression to distinguish TC cases from non-cancerous cases, was 0.6986 (Fig. 5). 
A

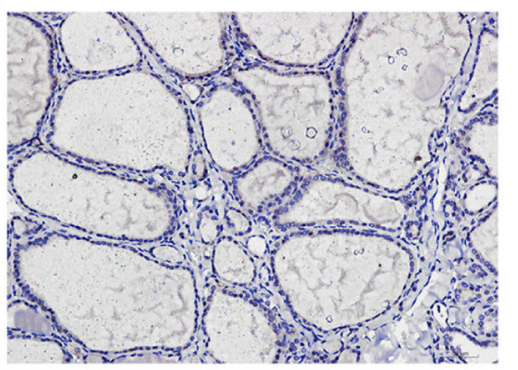

C

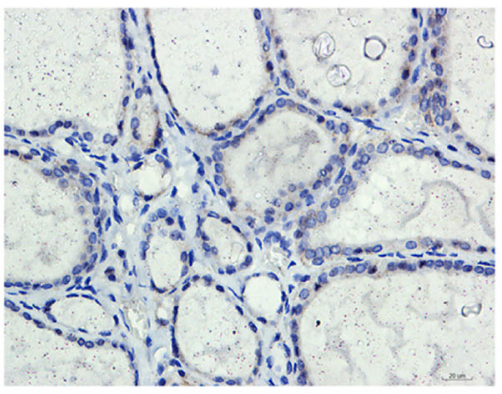

$\mathrm{E}$

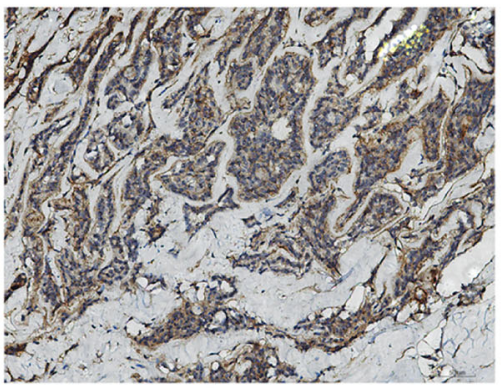

$\mathrm{G}$

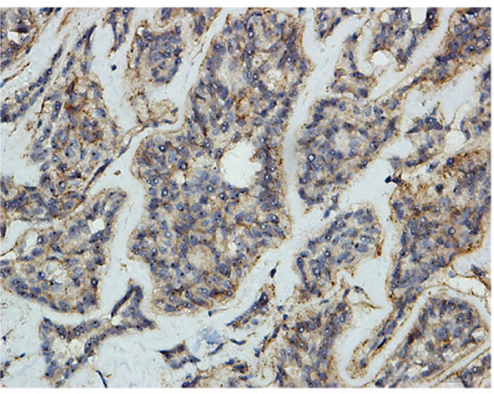

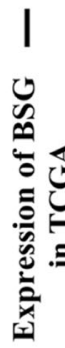

$\mathrm{P}<0.0001$

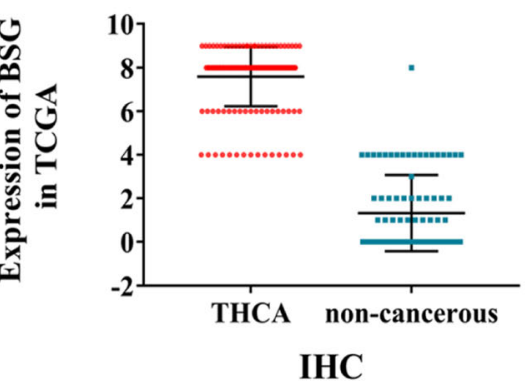

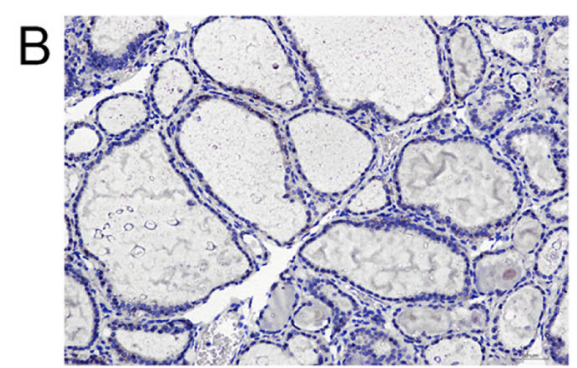

D

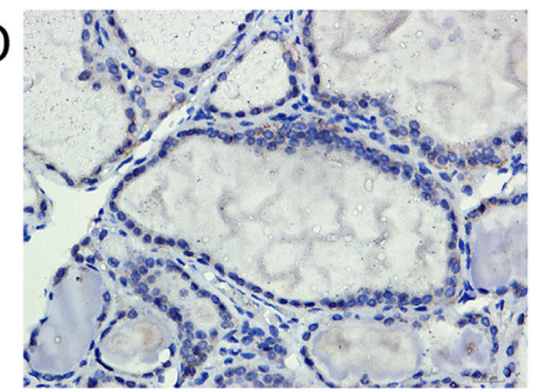

$F$
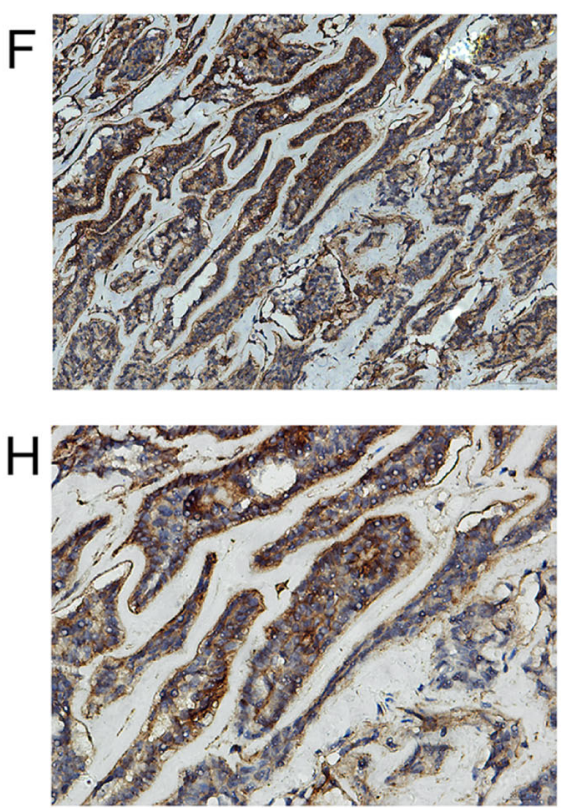

$\mathrm{J}$

IHC

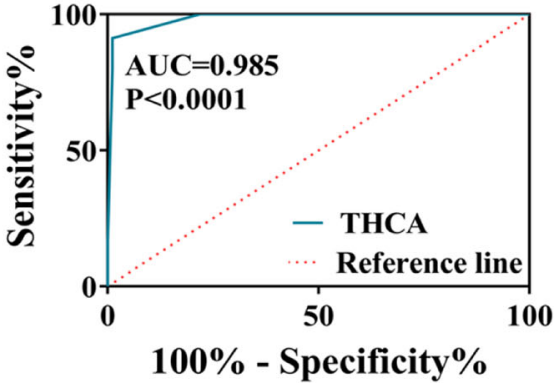

Fig. 1 (See legend on next page.) 
(See figure on previous page.)

Fig. 1 The results of BSG immunohistochemical staining. a BSG staining in non-cancer tissues $(\times 200)$. b BSG staining in non-cancer tissues $(x$ 200). c BSG staining in non-cancer tissues $(\times 400)$. d BSG staining in non-cancer tissues $(\times 400)$. e BSG staining in TC tissues $(\times 200)$. $\mathbf{f}$ BSG staining in TC tissues ( $\times 200)$. $\mathbf{g}$ BSG staining in TC tissues $(\times 400)$. $\mathbf{h}$ BSG staining in TC tissues ( $\times 400)$. $\mathbf{i}$ The expression of BSG in 171 TC and 87 noncancerous thyroid tissues. $\mathbf{j}$ The ROC curve was generated to assess the diagnostic ability of BSG in 171 TC and 87 non-cancerous thyroid tissues. The AUC was 0.985 (95\%Cl 0.971 to $0.999, p<0.0001)$, and the corresponding sensitivity and specificity was 0.912 and 0.966 , respectively

\section{Validation of BSG expression using CCLE cell coefficient data}

Using the CCLE database, we found that the expression levels of BSG in thyroid cell lines were higher than that in the majority of other types of cell lines (Fig. 6a). A heatmap of BSG expression in the TC cell lines was also constructed, and over half of the bands were red (Fig. 6b).

\section{The potential functions and pathways of BSG in TC}

We discovered 20092 BSG co-expressed genes using the cBioPortal for Cancer Genomics and 1447 DEGs using RRA methods. Finally, we discovered 1299 BSG co-expressed genes and DEGs by performing an intersection between the co-expressed gene data and the DEG data (Fig. 7a).

Table 1 Clinical pathological parameters and BSG expression in TC data from TCGA database

\begin{tabular}{|c|c|c|c|c|c|}
\hline \multirow[t]{2}{*}{ Characteristic } & & \multicolumn{4}{|c|}{ Expression of BSG $\left(\log _{2} x\right)$} \\
\hline & & $n$ & Mean \pm SD & t/F value & $p$ value \\
\hline \multirow[t]{2}{*}{ Tissue } & $\mathrm{TC}$ & 505 & $3.8 \pm 0.056$ & 6.647 & $<0.0001$ \\
\hline & Non-cancerous & 59 & $3.76 \pm 0.042$ & & \\
\hline \multirow[t]{2}{*}{ Gender } & Female & 369 & $3.7946 \pm 0.05313$ & 1.175 & 0.081 \\
\hline & Male & 136 & $3.8044 \pm 0.06241$ & & \\
\hline \multirow[t]{2}{*}{ Age } & $\geq 60$ & 120 & $3.8031 \pm 0.05706$ & -1.33 & 0.184 \\
\hline & $<60$ & 385 & $3.7954 \pm 0.05546$ & & \\
\hline \multirow[t]{5}{*}{ Pathologic T } & $\mathrm{T} 1$ & 143 & $3.7887 \pm 0.05514$ & 1.5 & 0.201 \\
\hline & $\mathrm{T} 2$ & 166 & $3.7991 \pm 0.05678$ & & \\
\hline & T3 & 171 & $3.8006 \pm 0.05538$ & & \\
\hline & T4 & 23 & $3.8128 \pm 0.04993$ & & \\
\hline & Tx & 2 & $3.7882 \pm 0.12413$ & & \\
\hline \multirow[t]{3}{*}{$\mathrm{T}$} & $\mathrm{T} 1-\mathrm{T} 2$ & 309 & $3.7943 \pm 0.05618$ & 1.17 & 0.311 \\
\hline & T3-T4 & 194 & $3.802 \pm 0.05479$ & & \\
\hline & Tx & 2 & $3.7882 \pm 0.12413$ & & \\
\hline \multirow[t]{3}{*}{ Pathologic N } & NO & 230 & $3.8047 \pm 0.06035$ & 4.387 & 0.013 \\
\hline & $\mathrm{N} 1$ & 225 & $3.7893 \pm 0.04527$ & & \\
\hline & Nx & 50 & $3.7985 \pm 0.07198$ & & \\
\hline \multirow[t]{3}{*}{ Pathologic M } & MO & 283 & $3.794 \pm 0.05303$ & 1.922 & 0.147 \\
\hline & M1 & 9 & $3.8248 \pm 0.05971$ & & \\
\hline & $M x$ & 213 & $3.8004 \pm 0.05911$ & & \\
\hline \multirow[t]{4}{*}{ Stage } & । & 285 & $3.7925 \pm 0.05688$ & 4.011 & 0.008 \\
\hline & $\|$ & 52 & $3.8195 \pm 0.06654$ & & \\
\hline & III & 113 & $3.7948 \pm 0.04918$ & & \\
\hline & IV & 55 & $3.8057 \pm 0.04748$ & & \\
\hline \multirow[t]{2}{*}{ Stage } & $|-| \mid$ & 337 & $3.7967 \pm 0.05918$ & -0.323 & 0.747 \\
\hline & III-IV & 168 & $3.7984 \pm 0.04876$ & & \\
\hline \multirow[t]{4}{*}{ Subtype } & Other, specify & 9 & $3.8297 \pm 0.06582$ & 14.593 & $<0.0001$ \\
\hline & Thyroid papillary carcinoma_classical/usual & 358 & $3.7883 \pm 0.04898$ & & \\
\hline & Thyroid papillary carcinoma_follicular & 102 & $3.8266 \pm 0.06996$ & & \\
\hline & Thyroid papillary carcinoma_tall cell & 36 & $3.7948 \pm 0.0421$ & & \\
\hline
\end{tabular}



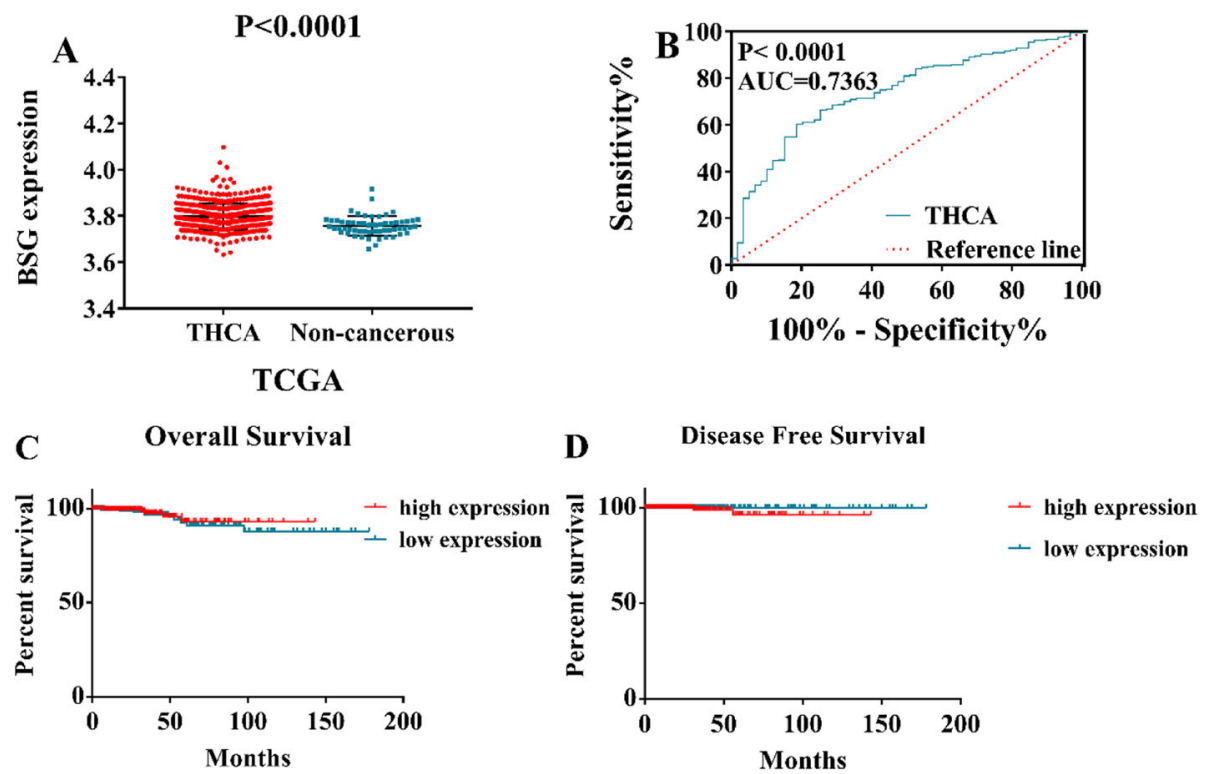

Fig. 2 Clinical role of upregulation of BSG mRNA level in thyroid cancer (TC) based on RNA-sequencing data. $\mathbf{a}$, $\mathbf{b}$ Expression level of BSG mRNA between TC and non-cancerous thyroid controls. c Overall survival, log-rank $p=0.5027$, hazard ratio (log-rank) $=0.6988(95 \% \mathrm{Cl} 0.2564$ to 1.905$)$. $\mathbf{d}$ Disease-free survival, log-rank $p=0.0947$, hazard ratio (Mantel-Haenszel) $=10.98$ (95\%Cl 0.6606 to 182.5)

Afterwards, GO and KEGG analyses were performed on these 1299 genes. The GO enrichment analysis unveiled enrichment of extracellular matrix (ECM) organization, cell adhesion, and inflammatory response in biological processes (BP). And we also discovered enrichment of extracellular space, proteinaceous extracellular matrix, and extracellular region in cellular component (CC). Furthermore, enrichment of calcium ion binding, heparin binding and extracellular matrix structural constituent were also found in molecular function (MF) (Fig. 7b-d, Table 3). Most importantly, the result of the KEGG analysis revealed that co-expressed genes and DEGs of BSG tended to enrich in the following terms: ECM-receptor interactions, complement and coagulation cascades, Staphylococcus aureus infection, p53 signaling pathway, and protein digestion and absorption (Fig. 7e, Table 3).

\section{Hub genes of BSG in TC}

Using PPI analysis and CytoHubba, we identified the top 10 genes as hub genes of BSG in TC (Fig. 8k): cyclin dependent kinase 1 (CDK1), kinesin family member 11 (KIF11), topoisomerase (DNA) II alpha (TOP2A), ribonucleotide reductase regulatory subunit M2 (RRM2), microtubule nucleation factor (TPX2), PDZ binding kinase (PBK), maternal embryonic leucine zipper kinase (MELK), DLG-associated protein 5 (DLGAP5), kinetochore complex component (NDC80), and nucleolar and spindle associated protein 1 (NUSAP1).

The expression levels of 9 hub genes in TC tissues were markedly higher than in non-cancerous tissues except KIF11: CDK1 (6.7486 \pm 0.70712 vs. $6.4577 \pm 0.86347, p<0.0001)$,
TOP2A (7.6083 \pm 1.19671 vs. $6.23 \pm 1.86183, p<0.0001)$, RRM2 (6.1999 \pm 1.25053 vs. $4.9689 \pm 2.35379)$, TPX2 $(6.5675 \pm 1.05049$ vs. $5.2402 \pm 1.88557, p<0.0001)$, PBK $(4.1448 \pm 1.18686$ vs. $2.9661 \pm 1.78316, p<0.0001)$, MELK $(4.422 \pm 1.27704$ vs. $2.8595 \pm 1.92385, p<0.0001)$, DLGAP5 (4.2636 \pm 1.36501 vs. $2.9592 \pm 2.27261, p<0.0001)$, NDC80 $(4.988 \pm 1.02333$ vs. $3.8061 \pm 1.70888, p<0.0001)$, and NUSAP1 (6.4232 \pm 1.00652 vs. $5.4004 \pm 1.67015, p<0.0001)$ (Fig. 8a-j, Supplementary Table S2). Corresponding AUCs for these hub genes were also obtained, including CDK1 $(\mathrm{AUC}=0.6571)$, TOP2A $(\mathrm{AUC}=0.741), \mathrm{RRM} 2(\mathrm{AUC}=$ 0.6911), TPX2 (AUC $=0.7392)$, PBK $($ AUC $=0.7275)$, MELK $(\mathrm{AUC}=0.753)$, DLGAP5 $(\mathrm{AUC}=0.7101), \mathrm{NDC} 80(\mathrm{AUC}=$ 0.738 ), and NUSAP1 (AUC $=0.7234$ ) (all $p<0.0001$ ) (Supplementary Figure S3, Supplementary Table S2).

\section{Discussion}

This study had two main aims. One was to identify the change of basigin (BSG) expression in thyroid cancer (TC) by collecting all possible sources of data, including data from IHC, RNA-seq, microarrays, and literature. The other was to create a preliminary study of the possible underlying mechanisms of BSG in TC using coexpressed genes and DEGs. We collected 1182 TC cases and 437 non-cancerous cases $(n=17)$ from all possible sources, and the combined SMD for BSG was $0.39(p<$ 0.0001). The AUC, which was used to assess the capacity of BSG to distinguish TC from non-cancerous tissues, was 0.6986. Subsequently, we found that BSG levels were upregulated in TC. We also performed GO and KEGG analyses for BSG co-expressed genes and DEGs, and the 

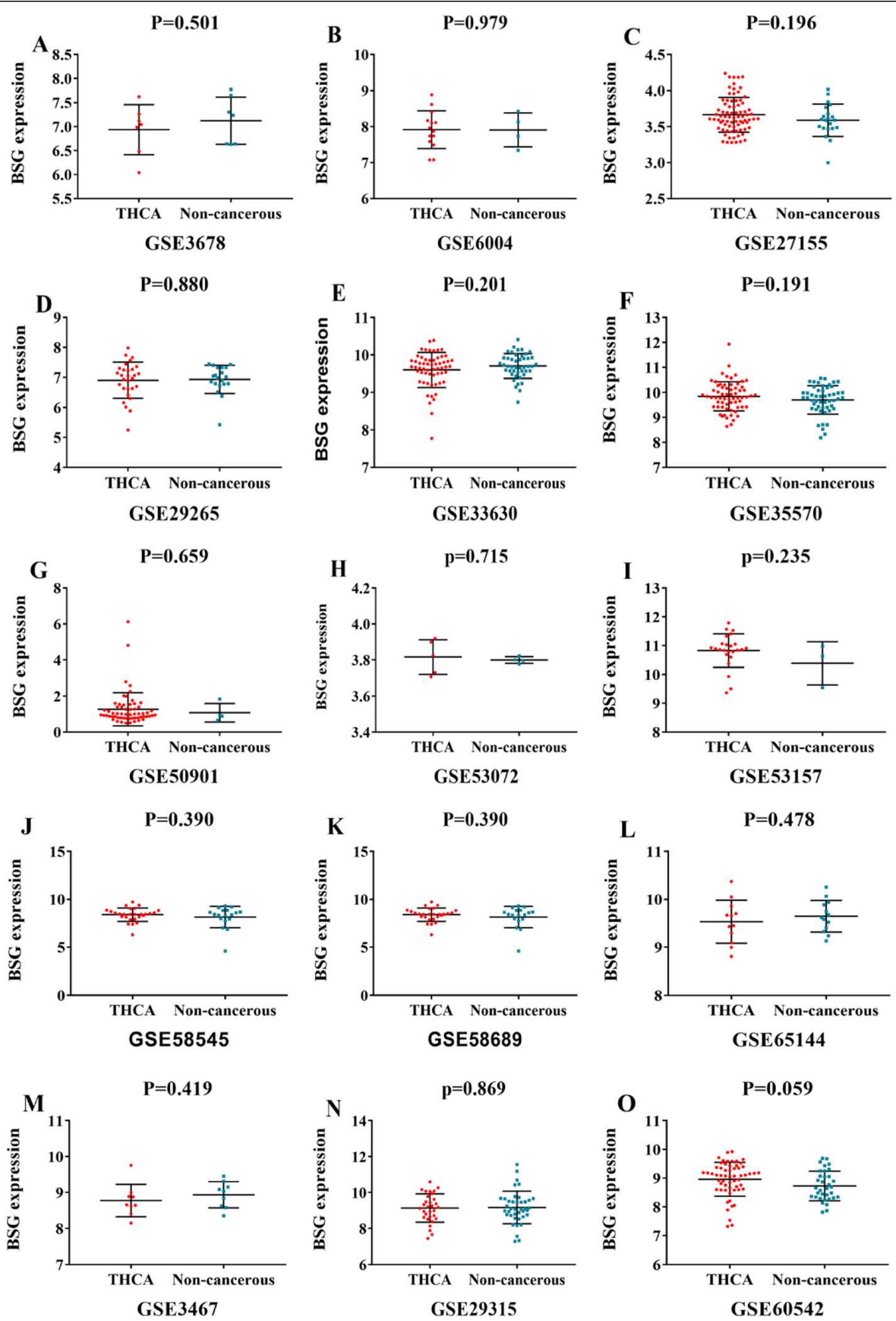

Fig. 3 (See legend on next page.) 
(See figure on previous page.)

Fig. 3 Scatter plots of BSG mRNA expression in thyroid cancer (TC) based on 15 GEO gene microarrays. a Microarray GSE3678. b

MicroarrayGSE6004. c Microarray GSE27155. d Microarray GSE29265. e Microarray GSE33630. f Microarray GSE35570. g Microarray GSE50901. h Microarray GSE53072. i Microarray GSE53751. j Microarray GSE58545. k Microarray GSE58689. I Microarray GSE65144. m Microarray GSE3467. n Microarray GSE29315. o Microarray GSE60542

results showed that genes tended to enrich in terms which were corrected with the extracellular matrix (ECM). Therefore, we surmised that BSG plays a part in TC by means of infecting the ECM.

As we all know, TC is the most common endocrine malignancy. Moreover, present researchers who have studied BSG expression levels have shown that BSG plays an important part in tumor cell invasion, metastasis, and angiogenesis [40-44]. Previous studies tended to focus on the relationship between BSG and differentiation levels of TC
$[16,17]$. Nevertheless, few studies have contributed to understanding the relationship between BSG expression levels and TC. In this study, we constructed an analysis using a large sample gathered from all possible sources, including co-expressed genes and DEGs of BSG, which have never been done before. We sincerely hope that the results of this study will assist with the development of TC therapy and provide a new direction for further study of BSG and TC.

Few studies have focused on the expression levels of the BSG protein and mRNA in TC, and previous studies

Table 2 Expression levels of BSG in the microarrays

\begin{tabular}{|c|c|c|c|c|c|c|}
\hline ID & Year & & $n$ & Mean \pm SD & $t$ value & $P$ value \\
\hline \multirow[t]{2}{*}{ GSE27155 } & 2011 & $\mathrm{TC}$ & 78 & $3.6650 \pm 0.2420$ & 1.302 & 0.196 \\
\hline & & Non-cancerous & 21 & $3.5886 \pm 0.2249$ & & \\
\hline \multirow[t]{2}{*}{ GSE53072 } & 2013 & $\mathrm{TC}$ & 5 & $3.8166 \pm 0.0956$ & 0.389 & 0.715 \\
\hline & & Non-cancerous & 4 & $3.7995 \pm 0.0188$ & & \\
\hline \multirow[t]{2}{*}{ GSE65144 } & 2015 & $\mathrm{TC}$ & 12 & $9.5326 \pm 0.4483$ & -0.722 & 0.478 \\
\hline & & Non-cancerous & 13 & $9.6457 \pm 0.3306$ & & \\
\hline \multirow[t]{2}{*}{ GSE29315 } & 2012 & $\mathrm{TC}$ & 31 & $9.1377 \pm 0.7861$ & -0.166 & 0.869 \\
\hline & & Non-cancerous & 40 & $9.1715 \pm 0.9021$ & & \\
\hline \multirow[t]{2}{*}{ GSE3678 } & 2006 & $\mathrm{TC}$ & 7 & $6.9376 \pm 0.251$ & -0.693 & 0.501 \\
\hline & & Non-cancerous & 7 & $7.1255 \pm 0.4929$ & & \\
\hline \multirow[t]{2}{*}{ GSE6004 } & 2006 & $\mathrm{TC}$ & 14 & $7.9174 \pm 0.5241$ & 0.027 & 0.979 \\
\hline & & Non-cancerous & 4 & $7.9096 \pm 0.4714$ & & \\
\hline \multirow[t]{2}{*}{ GSE29265 } & 2012 & $\mathrm{TC}$ & 29 & $6.9111 \pm 0.5993$ & -0.151 & 0.880 \\
\hline & & Non-cancerous & 20 & $6.9352 \pm 0.4666$ & & \\
\hline \multirow[t]{2}{*}{ GSE58545 } & 2015 & $\mathrm{TC}$ & 27 & $8.3943 \pm 0.7030$ & 0.868 & 0.390 \\
\hline & & Non-cancerous & 18 & $8.1597 \pm 1.1135$ & & \\
\hline \multirow[t]{2}{*}{ GSE58689 } & 2015 & $\mathrm{TC}$ & 27 & $8.3943 \pm 0.7030$ & 0.868 & 0.390 \\
\hline & & Non-cancerous & 18 & $8.1597 \pm 1.1135$ & & \\
\hline \multirow[t]{2}{*}{ GSE35570 } & 2015 & $\mathrm{TC}$ & 65 & $9.8489 \pm 0.5833$ & 1.315 & 0.191 \\
\hline & & Non-cancerous & 51 & $9.7066 \pm 0.5724$ & & \\
\hline \multirow[t]{2}{*}{ GSE33630 } & 2012 & $\mathrm{TC}$ & 59 & $9.6007 \pm 0.4657$ & -1.286 & 0.201 \\
\hline & & Non-cancerous & 45 & $9.7057 \pm 0.3300$ & & \\
\hline \multirow[t]{2}{*}{ GSE53157 } & 2013 & $\mathrm{TC}$ & 24 & $10.8278 \pm 0.5792$ & 1.218 & 0.235 \\
\hline & & Non-cancerous & 3 & $10.3846 \pm 0.7451$ & & \\
\hline \multirow[t]{2}{*}{ GSE50901 } & 2014 & $\mathrm{TC}$ & 61 & $1.3010 \pm 0.8936$ & 0.444 & 0.659 \\
\hline & & Non-cancerous & 4 & $1.0997 \pm 0.4919$ & & \\
\hline \multirow[t]{2}{*}{ GSE3467 } & 2005 & $\mathrm{TC}$ & 9 & $8.7789 \pm 0.4506$ & -0.829 & 0.419 \\
\hline & & Non-cancerous & 9 & $8.9390 \pm 0.3642$ & & \\
\hline \multirow[t]{2}{*}{ GSE60542 } & 2015 & $\mathrm{TC}$ & 58 & $8.9624 \pm 0.5898$ & 1.910 & 0.059 \\
\hline & & Non-cancerous & 34 & $8.7006 \pm 0.5161$ & & \\
\hline
\end{tabular}




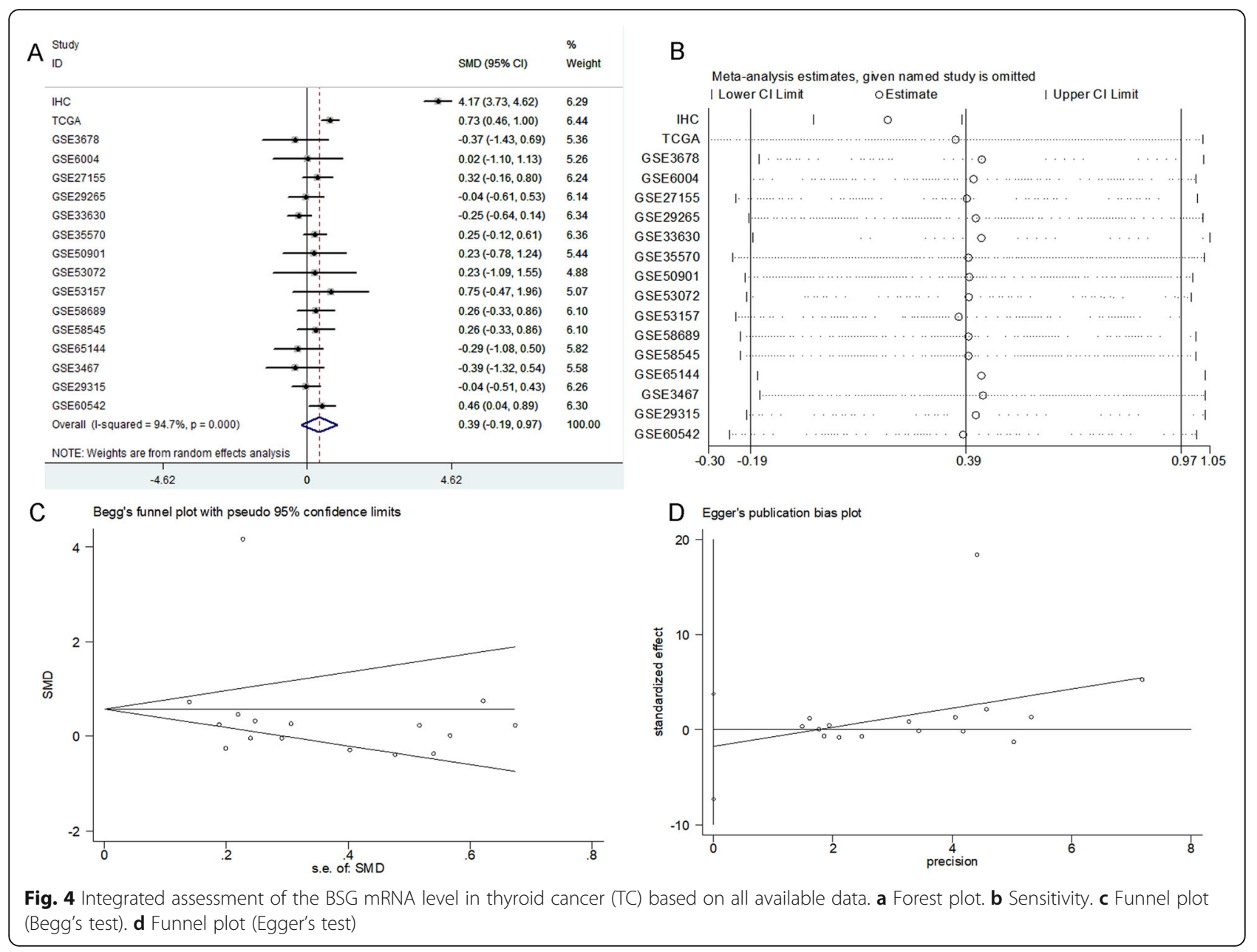

were more likely to focus on how the differentiation level of TC was linked with the positive expression of BSG and MMP. In addition, the sample sizes of these studies were small, and the methods were limited to IHC. Aratake et al. [16, 45] indicated that BSG expression correlated significantly with the degree of dedifferentiation of TC based on immunocytochemical analysis. More interestingly, they also observed MMP-2-positive expression in tumor cells and/or the adjacent tissue in all anaplastic carcinoma (ATC) cases, and the cytological atypia of cells with BSG positive expression was greater than that in cells with negative BSG expression. Tan et al. [20, 21] found that BSG and MMP-2 were expressed mainly in cancerous lesions by means of IHC staining of 156 differentiated carcinoma (DTC) cases. More importantly, they also observed that the positive expression of these two markers was more likely to be positively linked to extrathyroidal invasion, lymph node metastasis, depth of tumor invasion, and distant metastasis. This leads us to believe that the expression of BSG may be useful to predict the prognosis of DTC patients. Huang et al. $[18,46]$ evaluated the expression of BSG in
200 TC specimens and 20 nodular goiter specimens using IHC. They suggested that cytoplasmic BSG expression levels were significantly higher in TC tissues than in nodular goiter tissues and significantly higher across different pathological stages, and that they closely correlated with lymph node metastasis and depth of tumor invasion.

In the current study, we collected 171 cases of TC tissues and 87 cases of non-cancerous tissues from the First Affiliated Hospital of Guangxi Medical University and analyzed the BSG expression profiles. In doing so, we discovered that the protein expression levels of BSG were significantly higher in TC cases based on the IHC experiments. The AUC of BSG expression was 0.985, and the corresponding sensitivity and specificity were 0.912 and 0.966 , respectively. We also found that expression of the BSG protein tended to be statistically linked to cancer subtypes $(p<0.0001)$ based on the analysis of clinical parameters. However, the number of undifferentiated thyroid carcinomas (UTC) was only 2 . Therefore, the more specific relationship between BSG expression levels and differentiation levels of TC needs further 


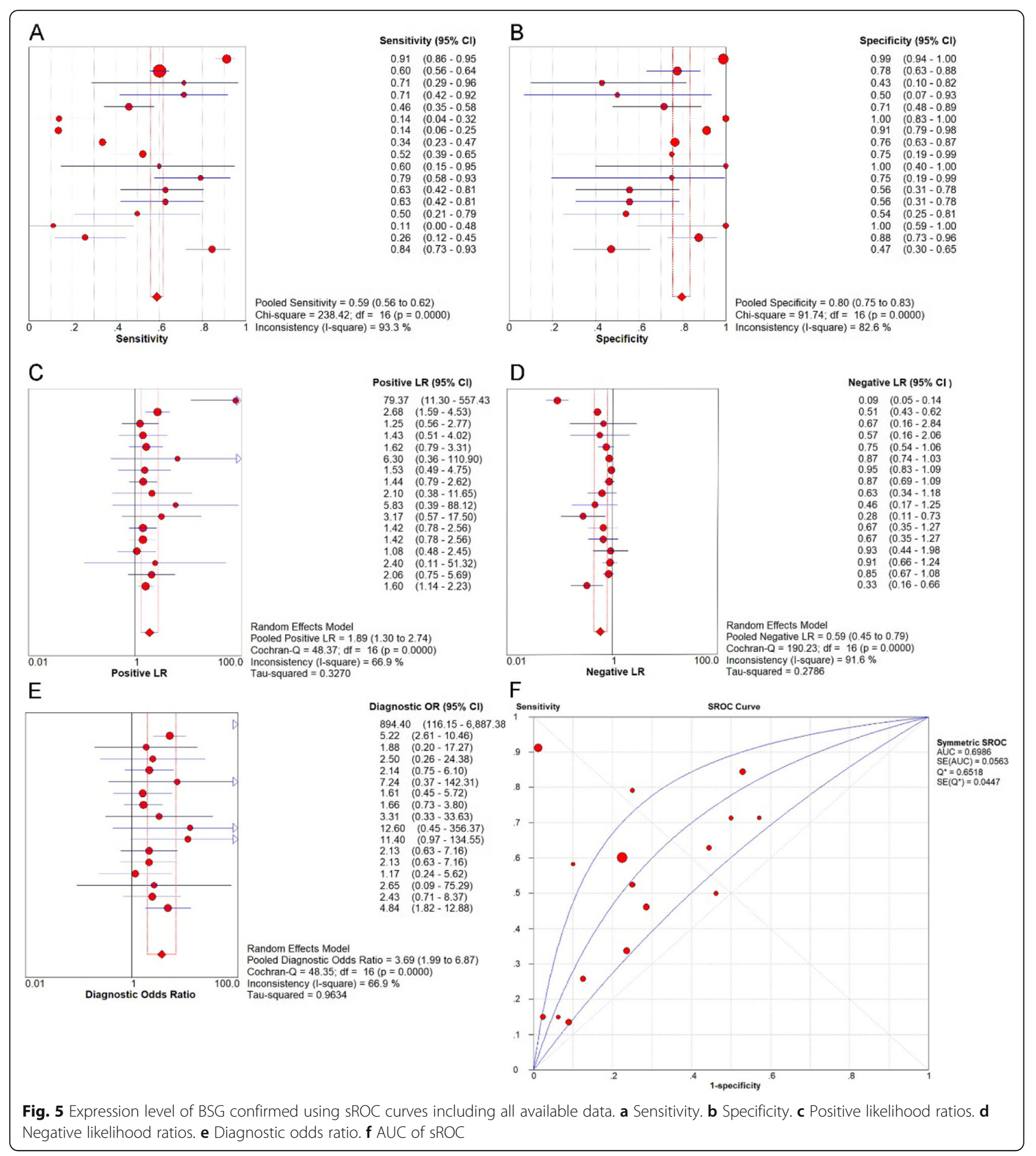

study and validation. More importantly, we also analyzed BSG expression profiles using 1182 TC cases and 437 non-cancerous cases $(n=17)$ collected from all possible sources, including IHC, RNA-seq, and microarrays. The combined SMD for BSG expression was 0.39, the diagnostic odds ratio was 3.69, and the AUC of the sROC curve was 0.6986. Making use of 505 TC cases from
RNA-seq based on the Kaplan-Meier survival curve analysis, we found that TC patients with high BSG expression levels tended to have slightly worse rates of diseasefree survival. However, the number of cases with BSG mRNA expression level and disease-free time was inadequate and accounted for a small proportion. Therefore, the survival analysis had a large bias. And the prognostic 

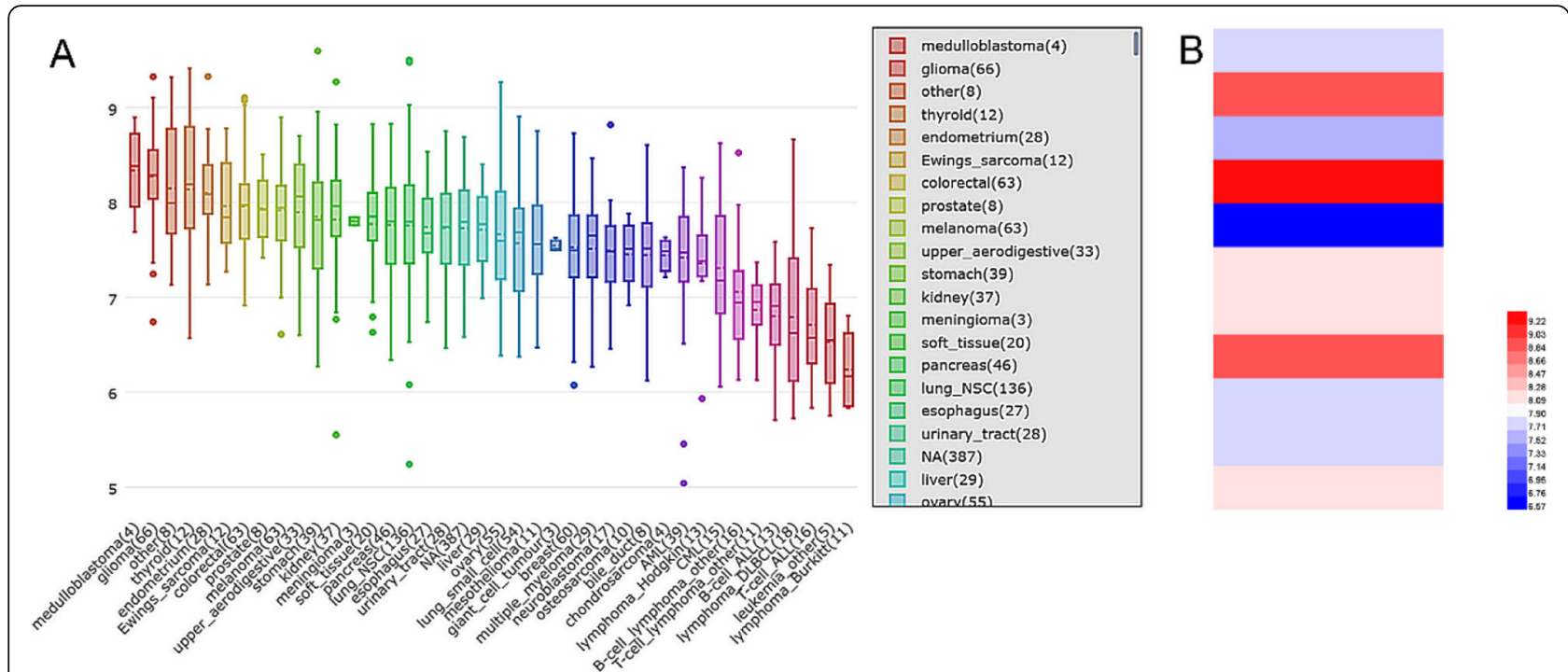

Fig. 6 Expression of BSG mRNA in thyroid cancer (TC) cell lines from the Cancer Cell Line Encyclopedia (CCLE). a Expression of BSG mRNA in all cell types. $\mathbf{b}$ Expression of BSG mRNA in all TC cell lines

ability of BSG to predict survival needs to be checked out by a large amount of cases and further validated.

Previous studies have shown that BSG played an important role in cancers, but which mechanisms it used to promote the occurrence and development of TC? A recent study [46] demonstrated that BSG was a cofactor of TNF receptor-associated factor 6 (TRAF6). Shi et al. [47] indicated that TRAF6 and BSG assisted with BSGmediated MMP9 could form the TRAF6/CD147/MMP9 pathway and was associated with angiogenesis and metastasis in ATC. Omi et al. [19] suggested the involvement of BSG in the invasiveness of follicular carcinoma (FTC) cells via regulation of MMPs based on immunoblot and IHC techniques. In addition, they indicated that upregulated expression of BSG in FTC was driven by epidermal growth factor (EGF) via the PI3K, ERK, and JNK pathways. Furthermore, a previous study [48] also found that the upregulated expression of BSG directly contributed to tumor angiogenesis by stimulating vascular endothelial growth factor (VEGF) production via the PI3K/AKT pathway. Li et al. [17] revealed that silencing of BSG in the TT cells in medullary carcinoma (MTC) could drive the inhibited proliferation of TT cells and the alteration of the cell cycle. Huang et al. [49] first confirmed that BSG took part in glycolysis and the transmembrane transport of lactate assisting with monocarboxylate transporters 1 (MCT1), a facilitator of lactate transport. This resulted in the decrease of extracellular $\mathrm{pH}$ and tumor progression. Fanelli et al. [50] revealed that Thyroid Stimulating Hormone (TSH) further upregulated the MCT/BSG complex, which was necessary for BSG expression, and assisted BSG translocation to the cytoplasmic membrane. In addition,
Huang et al. [18] indicated that miR-125a-5p functioned as a tumor suppressor in TC by directly targeting and repressing BSG expression, thereby inhibiting aerobic glycolysis and subsequently suppressing cancer cell viability, migration, and invasion.

In the present study, we constructed GO and KEGG clustering analyses of 1299 candidate BSG co-expressed genes and DEGs in TC. And these BSG co-expressed genes and DEGs tended to enrich in pathways which were associated with ECM, cell adhesion, cell-cell interactions, and angiogenesis. In the KEGG pathway analysis, genes were more likely enriched in the following terms: ECM-receptor interactions, complement and coagulation cascades, Staphylococcus aureus infections, the p53 signaling pathway, and in protein digestion and absorption. At the same time, the thyroid hormone synthesis pathways, pathways in cancers, and the PI3K-Akt signaling pathway were also evident. Due to these results, BSG, a member of the immunoglobulin superfamily, was determined to be a potential stimulator of MMP. It stimulated MMP-mediated ECM degradation, cell adhesion, and cell-cell interactions and played a crucial part in tumor cell invasion [9, 51-54]. BSG could also promote tumor angiogenesis via regulating MMPs and VEGF. Additionally, many previous researchers have studied the relationship between BSG and human cancers and further confirmed the role of BSG in the development of human tumors. For example, Li et al. [55] indicated that BSG promoted cell proliferation, metastasis, and invasion in breast cancer via accommodating the expression of MMP-9 and VEGF. Qiao et al. [43] revealed that BSG expression was significantly upregulated in malignant bone cancer. Moreover, the expression of 


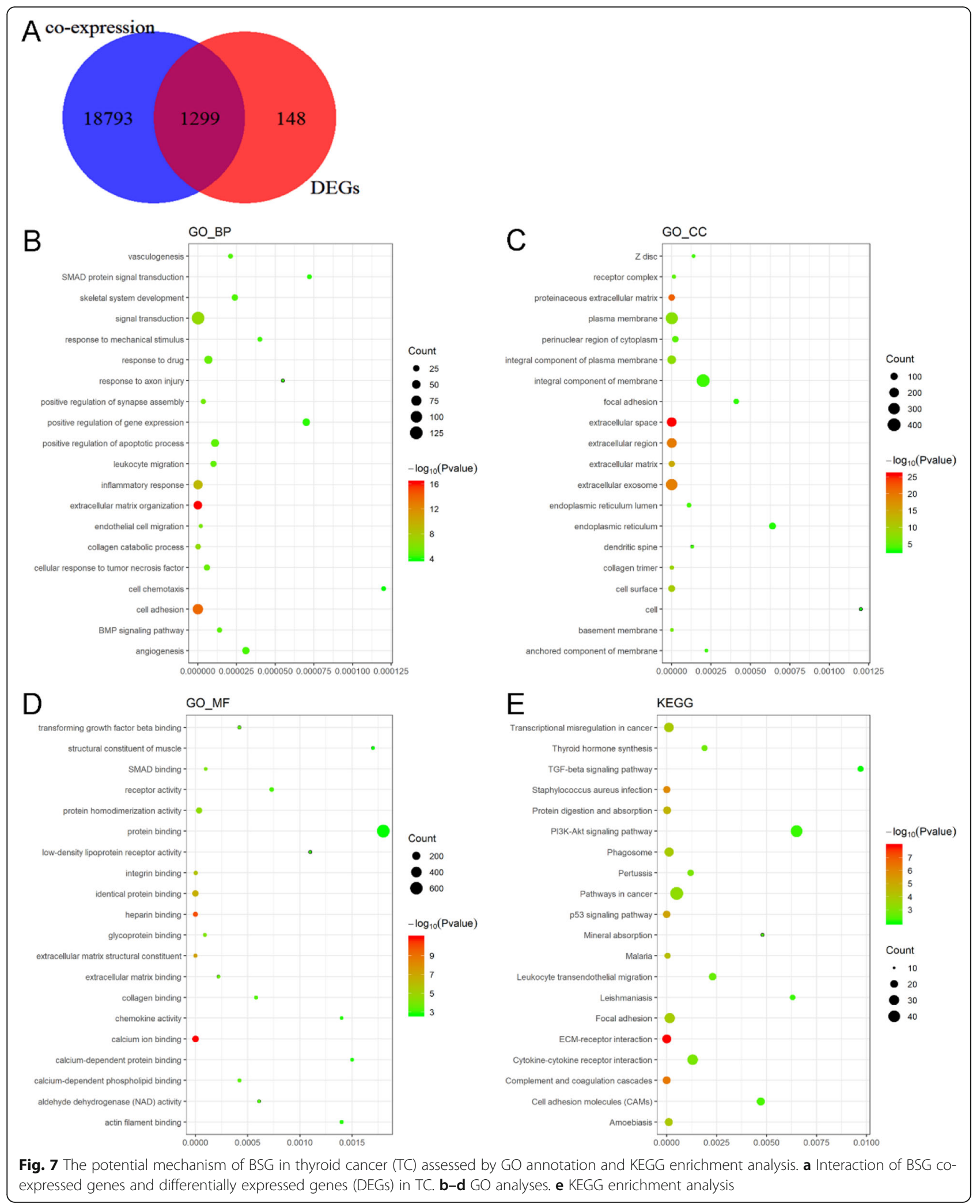


Table 3 Enrichment of functions and signaling pathways with BSG-related gene in thyroid cancer

\begin{tabular}{|c|c|c|c|c|}
\hline Category & Term & Count & $\%$ & $P$ value \\
\hline GO_BP & Extracellular matrix organization & 52 & 4 & $5.9 \mathrm{E}-17$ \\
\hline GO_BP & Cell adhesion & 81 & 6.2 & $1.4 \mathrm{E}-14$ \\
\hline GO_BP & Inflammatory response & 62 & 4.8 & $6 \mathrm{E}-10$ \\
\hline GO_BP & Signal transduction & 128 & 9.8 & $1.5 \mathrm{E}-07$ \\
\hline GO_BP & Collagen catabolic process & 19 & 1.5 & $2.2 \mathrm{E}-07$ \\
\hline GO_BP & Endothelial cell migration & 12 & 0.9 & $1.9 \mathrm{E}-06$ \\
\hline GO_BP & Positive regulation of synapse assembly & 17 & 1.3 & $3.5 \mathrm{E}-06$ \\
\hline GO_BP & Cellular response to tumor necrosis factor & 23 & 1.8 & $5.8 \mathrm{E}-06$ \\
\hline GO_BP & Response to drug & 44 & 3.4 & $6.9 \mathrm{E}-06$ \\
\hline GO_BP & Leukocyte migration & 24 & 1.8 & 0.00001 \\
\hline GO_BP & Positive regulation of apoptotic process & 43 & 3.3 & 0.000011 \\
\hline GO_BP & BMP signaling pathway & 18 & 1.4 & 0.000014 \\
\hline GO_BP & Vasculogenesis & 15 & 1.2 & 0.000021 \\
\hline GO_BP & Skeletal system development & 25 & 1.9 & 0.000024 \\
\hline GO_BP & Angiogenesis & 34 & 2.6 & 0.000031 \\
\hline GO_BP & Response to mechanical stimulus & 15 & 1.2 & 0.00004 \\
\hline GO_BP & Response to axon injury & 10 & 0.8 & 0.000055 \\
\hline GO_BP & Positive regulation of gene expression & 37 & 2.8 & 0.00007 \\
\hline GO_BP & SMAD protein signal transduction & 15 & 1.2 & 0.000072 \\
\hline GO_BP & Cell chemotaxis & 15 & 1.2 & 0.00012 \\
\hline GO_CC & Extracellular space & 199 & 15.3 & $1.9 \mathrm{E}-26$ \\
\hline GO_CC & Proteinaceous extracellular matrix & 70 & 5.4 & $1.3 \mathrm{E}-22$ \\
\hline GO_CC & Extracellular region & 208 & 16 & $2.4 \mathrm{E}-20$ \\
\hline GO_CC & Extracellular exosome & 311 & 23.9 & $6 \mathrm{E}-20$ \\
\hline GO_CC & Extracellular matrix & 62 & 4.8 & $4.5 \mathrm{E}-15$ \\
\hline GO_CC & Cell surface & 80 & 6.2 & $1 \mathrm{E}-10$ \\
\hline GO_CC & Collagen trimer & 26 & 2 & $1.5 \mathrm{E}-09$ \\
\hline GO_CC & Integral component of plasma membrane & 151 & 11.6 & $2.5 \mathrm{E}-08$ \\
\hline GO_CC & Plasma membrane & 361 & 27.8 & $4.4 \mathrm{E}-08$ \\
\hline GO_CC & Basement membrane & 20 & 1.5 & $1.1 \mathrm{E}-06$ \\
\hline GO_CC & Receptor complex & 24 & 1.8 & 0.000015 \\
\hline GO_CC & Perinuclear region of cytoplasm & 71 & 5.5 & 0.000023 \\
\hline GO_CC & Endoplasmic reticulum lumen & 29 & 2.2 & 0.00011 \\
\hline GO_CC & Dendritic spine & 19 & 1.5 & 0.00013 \\
\hline GO_CC & Z disk & 21 & 1.6 & 0.00014 \\
\hline GO_CC & Integral component of membrane & 409 & 31.5 & 0.0002 \\
\hline GO_CC & Anchored component of membrane & 20 & 1.5 & 0.00022 \\
\hline GO_CC & Focal adhesion & 46 & 3.5 & 0.00041 \\
\hline GO_CC & Endoplasmic reticulum & 82 & 6.3 & 0.00064 \\
\hline GO_CC & Cell & 17 & 1.3 & 0.0012 \\
\hline GO_MF & Calcium ion binding & 99 & 7.6 & $1.2 \mathrm{E}-11$ \\
\hline GO_MF & Heparin binding & 37 & 2.8 & $1 E-10$ \\
\hline GO_MF & Extracellular matrix structural constituent & 20 & 1.5 & $5.3 \mathrm{E}-08$ \\
\hline GO_MF & Identical protein binding & 89 & 6.8 & $2.1 \mathrm{E}-07$ \\
\hline
\end{tabular}


Table 3 Enrichment of functions and signaling pathways with BSG-related gene in thyroid cancer (Continued)

\begin{tabular}{|c|c|c|c|c|}
\hline Category & Term & Count & $\%$ & $P$ value \\
\hline GO_MF & Integrin binding & 23 & 1.8 & $1.6 \mathrm{E}-06$ \\
\hline GO_MF & Protein homodimerization activity & 79 & 6.1 & 0.000035 \\
\hline GO_MF & Glycoprotein binding & 15 & 1.2 & 0.000089 \\
\hline GO_MF & SMAD binding & 12 & 0.9 & 0.000098 \\
\hline GO_MF & Extracellular matrix binding & 9 & 0.7 & 0.00022 \\
\hline GO_MF & Transforming growth factor beta binding & 7 & 0.5 & 0.00042 \\
\hline GO_MF & Calcium-dependent phospholipid binding & 13 & 1 & 0.00042 \\
\hline GO_MF & Collagen binding & 13 & 1 & 0.00058 \\
\hline GO_MF & Aldehyde dehydrogenase (NAD) activity & 7 & 0.5 & 0.00061 \\
\hline GO_MF & Receptor activity & 29 & 2.2 & 0.00073 \\
\hline GO_MF & Low-density lipoprotein receptor activity & 6 & 0.5 & 0.0011 \\
\hline GO_MF & Chemokine activity & 11 & 0.8 & 0.0014 \\
\hline GO_MF & Actin filament binding & 20 & 1.5 & 0.0014 \\
\hline GO_MF & Calcium-dependent protein binding & 12 & 0.9 & 0.0015 \\
\hline GO_MF & Structural constituent of muscle & 10 & 0.8 & 0.0017 \\
\hline GO_MF & Protein binding & 642 & 49.4 & 0.0018 \\
\hline KEGG_PATHWAY & ECM-receptor interaction & 25 & 1.9 & $1.3 \mathrm{E}-08$ \\
\hline KEGG_PATHWAY & Complement and coagulation cascades & 20 & 1.5 & $4.5 \mathrm{E}-07$ \\
\hline KEGG_PATHWAY & Staphylococcus aureus infection & 17 & 1.3 & $1.2 \mathrm{E}-06$ \\
\hline KEGG_PATHWAY & p53 signaling pathway & 18 & 1.4 & $6.2 \mathrm{E}-06$ \\
\hline KEGG_PATHWAY & Protein digestion and absorption & 20 & 1.5 & 0.000022 \\
\hline KEGG_PATHWAY & Malaria & 14 & 1.1 & 0.000046 \\
\hline KEGG_PATHWAY & Amoebiasis & 21 & 1.6 & 0.0001 \\
\hline KEGG_PATHWAY & Transcriptional misregulation in cancer & 28 & 2.2 & 0.00012 \\
\hline KEGG_PATHWAY & Phagosome & 26 & 2 & 0.00013 \\
\hline KEGG_PATHWAY & Focal adhesion & 32 & 2.5 & 0.00015 \\
\hline KEGG_PATHWAY & Pathways in cancer & 49 & 3.8 & 0.0005 \\
\hline KEGG_PATHWAY & Pertussis & 15 & 1.2 & 0.0012 \\
\hline KEGG_PATHWAY & Cytokine-cytokine receptor interaction & 33 & 2.5 & 0.0013 \\
\hline KEGG_PATHWAY & Thyroid hormone synthesis & 14 & 1.1 & 0.0019 \\
\hline KEGG_PATHWAY & Leukocyte transendothelial migration & 19 & 1.5 & 0.0023 \\
\hline KEGG_PATHWAY & Cell adhesion molecules (CAMs) & 21 & 1.6 & 0.0047 \\
\hline KEGG_PATHWAY & Mineral absorption & 10 & 0.8 & 0.0048 \\
\hline KEGG_PATHWAY & Leishmaniasis & 13 & 1 & 0.0063 \\
\hline KEGG_PATHWAY & PI3K-Akt signaling pathway & 40 & 3.1 & 0.0065 \\
\hline KEGG_PATHWAY & TGF-beta signaling pathway & 14 & 1.1 & 0.0097 \\
\hline
\end{tabular}

TRACP, MMP-2, MMP-9, and c-Src (osteoclast specific genes) was analyzed by RT-PCR. In a word, BSG promoted the cancer cell invasion and development of TC via regulating MMPs and affecting ECM. This was also true of the PI3K-Akt signaling pathway.

The important role played by BSG in tumor cell invasion made this gene an excellent target for cancer treatment. At present, there are options for targeted therapy of TC. Based on the immune response to specific antigenic peptides present on the tumor surface or cytoplasm, several immunotherapeutic strategies have been developed. For instance, chimeric antigen receptor (CAR)-T cell therapy targeting intercellular adhesion molecule (ICAM)-1 was preclinically validated in TCs, especially in PTC and ATC [56]. Capdevila et al. demonstrated the effectiveness of blocking checkpoints with 


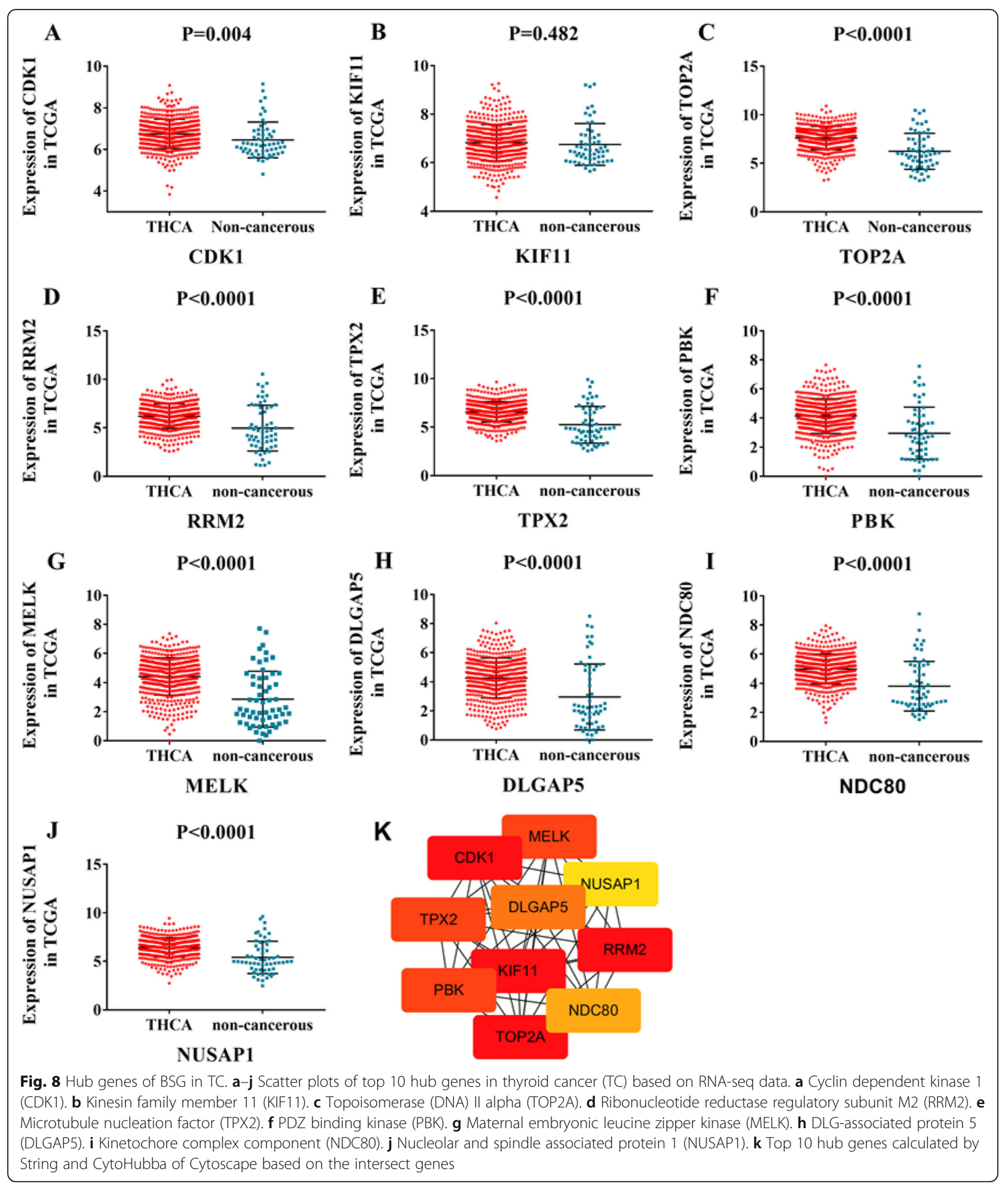

programmed death-1 (PD-1)/PD ligand 1 (PD-L1) axis inhibitors in ATC $[57,58]$. Cytotoxic T-lymphocyteassociated protein 4 (CTLA-4) could also be utilized in TC therapy. Furthermore, chemokine receptor-targeted immunotherapy for thyroid cancer was also worthy of attention, since previous studies have found that chemokines and their receptors such as CXCR1, CXCR2, CXCR3, CXCR4, CXCR7, DARC, CCR3, CCR6, CCR7, and CXCL5-CXCR2 axis play an important role in determining the cellular immunophenotype of thyroid tumor 
microenvironment [59]. Moreover, some novel drugs were utilized to TC therapy. For example, apatinib could induce apoptosis and autophagy of PTC cells through PI3K/Akt/ mTOR signaling pathway [60]. Previous studies have indicated that emodin inhibited the angiogenesis and metastasis of ATC by regulating TRAF6-mediated pathways, including the TRAF6/CD147/MMP9 pathway [47]. Some studies have provided evidence that downregulation of BSG via lentivirus vector-based RNAi decreased cell proliferation, matrigel invasion, and tumor formation in breast cancer, especially in MCF-7 breast cancer cells [61, 62]. Zhang et al. [63] provided evidence that chimeric antigen receptor $\mathrm{T}$ cells induced by doxycycline targeting BSG could be used in the treatment of liver cancer. Another study also found that doxycycline inhibited the proliferation of gallbladder cancer cells by downregulating the expression levels of BSG and induced an early apoptosis response in cancer cells [64]. In addition, Wang et al. [65] found that metuzumab could inhibit metastasis of esophageal cancer via blocking the function of BSG. Furthermore, BSG inhibitors are now being developed and tested [66-68], and we hope our study can provide some evidence for BSG targeted treatment of TC.

There were still some limitations in this study. First, only 11 TC cell lines were collected from the CCLE database. Second, the insufficiency of RT-qPCR analysis was also evident. Besides, the number of cases with BSG mRNA expression levels and disease-free time was limited making the survival analysis have a large bias. The current results of survival analysis need to be checked out. Most importantly, the specific mechanisms underlying BSG in $\mathrm{TC}$ need further validation in vitro and in vivo.

\section{Conclusion}

In this study, we demonstrated the upregulation of BSG expression in TC using meta-analysis and statistical analysis on the data collected from all possible sources, including IHC experiments, RNA-seq data, and microarray data. More importantly, we showed that BSG levels were closely correlated with the progression of TC may by affecting the ECM, cell adhesion, and cell-cell interactions. We hope further study can be performed to discover the specific molecular machinery of BSG that promotes the biological aggressiveness of TC

\section{Supplementary information}

Supplementary information accompanies this paper at https://doi.org/10. 1186/s12957-020-01975-9.

Additional file 1: Supplementary Figure S1. Integrated assessment of the BSG mRNA level in thyroid cancer (THCA) based on gene microarray. A: Forest plot; B: Sensitivity; C: Funnel plot (Begg's test); D: Funnel plot (Egger's test). Supplementary Figure S2. Expression level of BSG confirmed using sROC curves using microarray data. A: Sensitivity; B: Specificity; C: Positive likelihood ratios; D: Negative likelihood ratios; E:
Diagnostic odds ratio; F: AUC of sROC. Supplementary Figure S3. Roc curve of top 10 hub genes in thyroid cancer (THCA) based on RNA-seq data. A: Cyclin dependent kinase 1 (CDK1); B: kinesin family member 11 (KIF11); C: topoisomerase (DNA) II alpha (TOP2A); D: ribonucleotide reductase regulatory subunit M2 (RRM2); E: microtubule nucleation factor (TPX2); F: PDZ binding kinase (PBK); G: maternal embryonic leucine zipper kinase (MELK); H: DLG associated protein 5 (DLGAP5); l: kinetochore complex component (NDC80); J: and nucleolar and spindle associated protein 1 (NUSAP1). Supplementary Table S1. Clinical pathological parameters and BSG expression in THCA data from IHC. Supplementary Table 2. The scores and expression of Top 10 hub genes in THCA

\section{Abbreviations}

ATC: Anaplastic carcinoma; AUC: Area under the curve; CAR: Chimeric antigen receptor; CCLE: Cancer Cell Line Encyclopedia; Cl: Confidential interval; CTLA-4: Cytotoxic T-lymphocyte-associated protein 4; DAVI D: Database for Annotation, Visualization, and Integrated Discovery; DEGs: Differentially expressed genes; DOR: Diagnostic odds ratio;

DTC: Differentiated carcinoma; ECM: Extracellular matrix; FTC: Follicular carcinoma; GEO: Gene Expression omnibus; GO: Gene Ontology; GO_BP: GO biological process; GO_CC: GO cellular component; GO_MF: GO molecular function; HCC: Hepatocellular carcinoma; IHC: Immunohistochemistry; KEGG: Kyoto Encyclopedia of Genes and Genomes; MMPs: Matrix metalloproteinases; MTC: Medullary carcinoma; NDC80: Kinetochore complex component; PD-1: Programmed death-1; PD-L1: PD ligand 1; PPI: Proteinprotein interaction; PTC: Papillary carcinoma; RNA-seq: RNA-sequencing; RTqPCR: Real-time quantitative polymerase chain reaction; SMD: Standard mean difference; SD: Standard deviation; SRA: Sequence Read Archive; SROC: Summary receiver operating characteristic; SP/NK-1R: Substance P/ neurokinin-1 receptor; STRING: Search Tool for the Retrieval of Interacting Genes; TCGA: The Cancer Genome Atlas; TC: Thyroid cancer; TSH: Thyroid stimulating hormone; UTC: Undifferentiated thyroid carcinoma; VEGF: Vascular endothelial growth factor

\section{Acknowledgements}

Not applicable.

\section{Authors' contributions}

G Chen, YY Pang, ZG Huang, XZ Tang, ZX Wei, and W Ma designed the study. WP Guo, QQ Lai, and JY Gan collected and analyzed the data. WP Guo, XL Huang, and XF Liu wrote the manuscript. G Chen, ZG Huang, XJ Li, D Tang, and W Ma revised the paper. All authors read and approved the final manuscript.

\section{Funding}

The Funds of Guangxi Zhuang Autonomous Region Health and Wellness Committee Science and Technology Project (S201415-06); Natural Science Foundation of Guangxi, China (Guikegong0861003); Natural Science Foundation of Guangxi, China (2017GXNSFAA198287); Guangxi Zhuang Autonomous Region Health and Family Planning Commission Self-Financed Scientific Research Project (Z2012053); Guangxi Degree and Postgraduate Education Reform and Development Research Projects, China (JGY2019050); Guangxi Medical University Education and Teaching Reform Project (2019XJGZ04); Guangxi Zhuang Autonomous Region Health and Family Planning Commission Self-financed Scientific Research Project (Z20180979); and Guangxi Zhuang Autonomous Region University Student Innovative Plan (No. 201910598148).

\section{Availability of data and materials}

The datasets generated and/or analyzed during the current study are available in the TCGA repository, https://cancergenome.nih.gov/, and the GEO repository, https://www.ncbi.nlm.nih.gov/geo/. The IHC data that support the findings of this study are available from Fanpu Biotech, Inc. (Guilin, China) but restrictions apply to the availability of these data, which were used under license for the current study, and so are not publicly available. Data are however available from the authors upon reasonable request and with permission of Fanpu Biotech, Inc. (Guilin, China). 


\section{Ethics approval and consent to participate}

Approval for the study was obtained from the Ethics Committee of the First Affiliated Hospital of Guangxi Medical University [Fanpu (No. 23 in 2018)]. Informed consent was obtained from all the patients whose tissues were used in this study.

\section{Consent for publication}

Not applicable.

\section{Competing interests}

The authors declare that they have no competing interests.

\section{Author details}

'Department of Pathology, First Affiliated Hospital of Guangxi Medical University, 6 Shuangyong Road, Nanning 530021, Guangxi Zhuang Autonomous Region, People's Republic of China. ${ }^{2}$ Department of Nuclear Medicine, First Affiliated Hospital of Guangxi Medical University, 6 Shuangyong Road, Nanning 530021, Guangxi Zhuang Autonomous Region, People's Republic of China. ${ }^{3}$ Department of Head and Neck Tumor Surgery, Guangxi Medical University Cancer Hospital, 71 Hedi Road, Nanning 530021, Guangxi Zhuang Autonomous Region, People's Republic of China.

Received: 19 April 2020 Accepted: 28 July 2020

Published online: 05 September 2020

\section{References}

1. Khatami F, Payab M, Sarvari M, Gilany K, Larijani B, Arjmand B, Tavangar SM. Oncometabolites as biomarkers in thyroid cancer: a systematic review. Cancer Manag Res. 2019;11:1829-41.

2. Lin P, Guo YN, Shi L, Li XJ, Yang H, He Y, Li Q, Dang YW, Wei KL, Chen G. Development of a prognostic index based on an immunogenomic landscape analysis of papillary thyroid cancer. Aging (Albany NY). 2019;11: 480-500.

3. Wang L, Zhang L, Zhu M, Qi X, Yi Z. Automatic diagnosis for thyroid nodules in ultrasound images by deep neural networks. Med Image Anal. 2020:61:101665.

4. Chen F, Yin S, Zhu J, Liu P, Yang C, Feng Z, Deng Z. IncRNA DGCR5 acts as a tumor suppressor in papillary thyroid carcinoma via sequestering miR2861. Exp Ther Med. 2019;17:895-900.

5. Han J, Zhang M, Nie C, Jia J, Wang F, Yu J, Bi W, Liu B, Sheng R, He G, et al. miR-215 suppresses papillary thyroid cancer proliferation, migration, and invasion through the AKT/GSK-3beta/Snail signaling by targeting ARFGEF1. Cell Death Dis. 2019:10:195.

6. Ji L, Fan X, Zhou F, Gu J, Deng X. IncRNA RPL34-AS1 inhibits cell proliferation and invasion while promoting apoptosis by competitively binding miR-3663-3p/RGS4 in papillary thyroid cancer. J Cell Physiol. 2020; 235:3669-78.

7. Zhuang X, Tong H, Ding Y, Wu L, Cai J, Si Y, Zhang H, Shen M. Long noncoding RNA ABHD11-AS1 functions as a competing endogenous RNA to regulate papillary thyroid cancer progression by miR-199a-5p/SLC1A5 axis. Cell Death Dis. 2019;10:620.

8. Isorna I, Esteban F, Solanellas J, Covenas R, Munoz M. The substance P and neurokinin-1 receptor system in human thyroid cancer: an immunohistochemical study. Eur J Histochem. 2020:64.

9. Kumar D, Vetrivel U, Parameswaran S, Subramanian KK. Structural insights on druggable hotspots in CD147: A bull's eye view. Life Sci. 2019;224:76-87.

10. Ames S, Andring JT, MC Kenna R, Becker HM. CAIX forms a transport metabolon with monocarboxylate transporters in human breast cancer cells. Oncogene. 2019.

11. Fan X, Wu W, Shi H, Han J. RNA interference targeting CD147 inhibits the invasion of human cervical squamous carcinoma cells by downregulating MMP-9. Cell Biol Int. 2013;37:737-41.

12. Guo W, Abudumijiti H, Xu L, Hasim A. CD147 promotes cervical cancer migration and invasion by up-regulating fatty acid synthase expression. Int J Clin Exp Pathol. 2019;12:4280-8.

13. Huang HK, Lee SY, Huang SF, Lin YS, Chao SC, Huang SF, Lee SC, Cheng TH, Loh SH, Tsai YT. Isoorientin decreases cell migration via decreasing functional activity and molecular expression of proton-linked monocarboxylate transporters in human lung cancer cells. Am J Chin Med. 2020;48:201-22.
14. Lucchetti D, Colella F, Perelli L, Ricciardi-Tenore C, Calapa F, Fiori ME, Carbone F, De Maria R, Sgambato A. CD147 promotes cell small extracellular vesicles release during colon cancer stem cells differentiation and triggers cellular changes in recipient cells. Cancers (Basel). 2020;12.

15. Xiong L, Edwards CK 3rd, Zhou L. The biological function and clinical utilization of CD147 in human diseases: a review of the current scientific literature. Int J Mol Sci. 2014;15:17411-41.

16. Aratake $Y$, Marutsuka K, Kiyoyama K, Kuribayashi T, Miyamoto T, Yakushiji K, Ohno S, Miyake Y, Sakaguchi T, Kobayashi TK, et al. EMMP RIN (CD147) expression and differentiation of papillary thyroid carcinoma: implications for immunocytochemistry in FNA cytology. Cytopathology. 2010;21:103-10.

17. Li J, Li D, Zhang L, Huang P, Li Z. Effects of CD147 gene silencing on protein expression of ANXA2, MMP-2 and TIMP-2 by thyroid medullary carcinoma TT cells and biologic characteristics. Zhonghua Bing Li Xue Za Zhi. 2014;43:103-8.

18. Huang P, Mao LF, Zhang ZP, Lv WW, Feng XP, Liao HJ, Dong C, Kaluba B, Tang XF, Chang S. Down-regulated miR-125a-5p promotes the reprogramming of glucose metabolism and cell malignancy by increasing levels of CD147 in thyroid cancer. Thyroid. 2018;28:613-23.

19. Omi Y, Shibata N, Okamoto T, Obara T, Kobayashi M. The role of CD147 in the invasiveness of follicular thyroid carcinoma cells. Thyroid. 2012;22:383-94.

20. Tan H, Ye K, Wang Z, Tang H. CD147 expression as a significant prognostic factor in differentiated thyroid carcinoma. Transl Res. 2008;152:143-9.

21. Tan H, Ye K, Wang Z, Tang H. Clinicopathologic evaluation of immunohistochemical CD147 and MMP-2 expression in differentiated thyroid carcinoma. Jpn J Clin Oncol. 2008;38:528-33.

22. Kleih M, Bopple K, Dong M, Gaissler A, Heine S, Olayioye MA, Aulitzky WE, Essmann F. Direct impact of cisplatin on mitochondria induces ROS production that dictates cell fate of ovarian cancer cells. Cell Death Dis. 2019;10:851

23. Ni X, Lin Z, Dai S, Chen H, Chen J, Zheng C, Wu B, Ao J, Shi K, Sun H. Screening and verification of microRNA promoter methylation sites in hepatocellular carcinoma. J Cell Biochem. 2020.

24. Qiu Y, Pu C, Li Y, Qi B. Construction of a circRNA-miRNA-mRNA network based on competitive endogenous RNA reveals the function of circRNAs in osteosarcoma. Cancer Cell Int. 2020;20:48.

25. Jing QB, Tong HX, Tang WJ, Tian SD. Clinical significance and potential regulatory mechanisms of serum response factor in 1118 cases of thyroid cancer based on gene chip and RNA-sequencing data. Med Sci Monit. 2020;26:e919302.

26. Wang L, Qu J, Liang Y, Zhao D, Rehman FU, Qin K, Zhang X. Identification and validation of key genes with prognostic value in non-small-cell lung cancer via integrated bioinformatics analysis. Thorac Cancer. 2020.

27. Wu HY, Xia S, Liu AG, Wei MD, Chen ZB, Li YX, He Y, Liao MJ, Hu QP, Pan SL. Upregulation of miR1323p in cholangiocarcinoma tissues: a study based on RTgPCR, The Cancer Genome Atlas miRNA sequencing, Gene Expression Omnibus microarray data and bioinformatics analyses. Mol Med Rep. 2019; 20:5002-20

28. Zhang L, Luo B, Dang YW, He RQ, Peng ZG, Chen G, Feng ZB. Clinical significance of microRNA-196b-5p in hepatocellular carcinoma and its potential molecular mechanism. J Cancer. 2019;10:5355-70.

29. Zheng HP, Huang ZG, He RQ, Lu HP, Dang YW, Lin P, Wen DY, Qin YY, Luo $B$, Li XJ, et al. Integrated assessment of CDK1 upregulation in thyroid cancer. Am J Transl Res. 2019;11:7233-54.

30. Zhong F, Lu HP, Chen G, Dang YW, Li GS, Chen XY, Qin YY, Yao YX, Zhang $X G$, Liang $Y$, et al. The clinical significance and potential molecular mechanism of integrin subunit beta 4 in laryngeal squamous cell carcinoma. Pathol Res Pract. 2020;216:152785.

31. Nusinow DP, Szpyt J, Ghandi M, Rose CM, McDonald ER, 3rd, Kalocsay M, Jane-Valbuena J, Gelfand E, Schweppe DK, Jedrychowski M, et al: Quantitative proteomics of the cancer cell line encyclopedia. Cell 2020, 180: 387-402 e316.

32. Shi K, Zhu X, Liu Z, Sun N, Gu L, Wei Y, Cheng X, Zhang Z, Xie B, Yang S, et al. Clinical characteristics of malignant melanoma in central China and predictors of metastasis. Oncol Lett. 2020;19:1452-64.

33. Dimitrakopoulos C, Hindupur SK, Hafliger L, Behr J, Montazeri H, Hall MN, Beerenwinkel N. Network-based integration of multi-omics data for prioritizing cancer genes. Bioinformatics. 2018;34:2441-8.

34. Guan YJ, Ma JY, Song W. Identification of circRNA-miRNA-mRNA regulatory network in gastric cancer by analysis of microarray data. Cancer Cell Int. 2019;19:183. 
35. Song ZY, Chao F, Zhuo Z, Ma Z, Li W, Chen G. Identification of hub genes in prostate cancer using robust rank aggregation and weighted gene coexpression network analysis. Aging (Albany NY). 2019;11:4736-56.

36. Emdad L, Bhoopathi P, Talukdar S, Pradhan AK, Sarkar D, Wang XY, Das SK, Fisher PB. Recent insights into apoptosis and toxic autophagy: The roles of MDA-7/IL-24, a multidimensional anti-cancer therapeutic. Semin Cancer Biol. 2019.

37. Xiao H, Xu D, Chen P, Zeng G, Wang X, Zhang X. Identification of five genes as a potential biomarker for predicting progress and prognosis in adrenocortical carcinoma. J Cancer. 2018;9:4484-95.

38. Liu X, Hou X, Zhou Y, Li Q, Kong F, Yan S, Lei S, Xiong L, He J. Downregulation of the helicase lymphoid-specific (HELLS) gene impairs cell proliferation and induces cell cycle arrest in colorectal cancer cells. Onco Targets Ther. 2019;12:10153-63.

39. Chin $\mathrm{CH}$, Chen $\mathrm{SH}, \mathrm{Wu} \mathrm{HH}, \mathrm{Ho} \mathrm{CW}, \mathrm{Ko} \mathrm{MT}$, Lin CY. cytoHubba: identifying hub objects and sub-networks from complex interactome. BMC Syst Biol. 2014;8(Suppl 4):S11.

40. Huang WT, Yang X, He RQ, Ma J, Hu XH, Mo WJ, Chen G. Overexpressed BSG related to the progression of lung adenocarcinoma with highthroughput data-mining, immunohistochemistry, in vitro validation and in silico investigation. Am J Transl Res. 2019;11:4835-50.

41. Jin J, Wang SJ, Cui J, Li L, Li JY, Liu FL, Sun XX, Jiang JL, Cui HY, Chen ZN. Hypo-phosphorylated CD147 promotes migration and invasion of hepatocellular carcinoma cells and predicts a poor prognosis. Cell Oncol (Dordr). 2019;42:537-54.

42. Liu J, Liu Q, Wang Y, Liu M, Qi Y, Gao J, Lin B. Coexpression of Lewis y antigen and CD147 in epithelial ovarian cancer is correlated with malignant progression and poor prognosis. Int J Mol Med. 2019;43:1687-98.

43. Qiao S, Liu C, Xu W, AZ W, Li C, Wang Z. Up-regulated expression of CD147 gene in malignant bone tumor and the possible induction mechanism during osteoclast formation. Braz J Med Biol Res. 2018;51:e6948.

44. Wang C, Zhang J, Fok KL, Tsang LL, Ye M, Liu J, Li F, Zhao AZ, Chan HC, Chen H. CD147 induces epithelial-to-mesenchymal transition by disassembling cellular apoptosis susceptibility protein/E-cadherin/betacatenin complex in human endometriosis. Am J Pathol. 2018;188:1597-607.

45. Aratake Y, Nomura H, Kotani T, Marutsuka K, Kobayashi K, Kuma K, Miyauchi A, Okayama A, Tamura K. Coexistent anaplastic and differentiated thyroid carcinoma: an immunohistochemical study. Am J Clin Pathol. 2006;125:399_ 406.

46. Luo Z, Zhang X, Zeng W, Su J, Yang K, Lu L, Lim CB, Tang W, Wu L, Zhao S, et al. TRAF6 regulates melanoma invasion and metastasis through ubiquitination of basigin. Oncotarget. 2016;7:7179-92.

47. Shi GH, Zhou L. Emodin suppresses angiogenesis and metastasis in anaplastic thyroid cancer by affecting TRAF6mediated pathways in vivo and in vitro. Mol Med Rep. 2018;18:5191-7.

48. Tang Y, Nakada MT, Rafferty P, Laraio J, McCabe FL, Millar H, Cunningham M, Snyder LA, Bugelski P, Yan L. Regulation of vascular endothelial growth factor expression by EMMPRIN via the PI3K-Akt signaling pathway. Mol Cancer Res. 2006;4:371-7

49. Huang P, Chang S, Jiang X, Su J, Dong C, Liu X, Yuan Z, Zhang Z, Liao H. RNA interference targeting CD147 inhibits the proliferation, invasiveness, and metastatic activity of thyroid carcinoma cells by down-regulating glycolysis. Int J Clin Exp Pathol. 2015;8:309-18.

50. Fanelli A, Grollman EF, Wang D, Philp NJ. MCT1 and its accessory protein CD147 are differentially regulated by TSH in rat thyroid cells. Am J Physiol Endocrinol Metab. 2003;285:E1223-9.

51. Albrechtsen R, Wewer Albrechtsen NJ, Gnosa S, Schwarz J, Dyrskjot L, Kveiborg M. Identification of ADAM12 as a novel basigin sheddase. Int J Mol Sci. 2019;20.

52. Guindolet D, Gabison EE. Role of CD147 (EMMPRIN/Basigin) in tissue remodeling. Anat Rec (Hoboken). 2019.

53. Xie Y, Wang Y, Ding H, Guo M, Wang X, Dong Q, Cui M. Highly glycosylated CD147 promotes hemorrhagic transformation after rt-PA treatment in diabetes: a novel therapeutic target? J Neuroinflammation. 2019;16:72.

54. Zhang Z, Yang X, Zhang H, Liu X, Pan S, Li C. The role of extracellular matrix metalloproteinase inducer glycosylation in regulating matrix metalloproteinases in periodontitis. J Periodontal Res. 2018;53:391-402.

55. Li F, Zhang J, Guo J, Jia Y, Han Y, Wang Z. RNA interference targeting CD147 inhibits metastasis and invasion of human breast cancer MCF-7 cells by downregulating MMP-9NEGF expression. Acta Biochim Biophys Sin (Shanghai). 2018;50:676-84.
56. Yin H, Tang Y, Guo Y, Wen S. Immune microenvironment of thyroid cancer. J Cancer. 2020;11:4884-96.

57. Ferrari SM, Elia G, Ragusa F, Ruffilli I, La Motta C, Paparo SR, Patrizio A, Vita R, Benvenga S, Materazzi G, et al. Novel treatments for anaplastic thyroid carcinoma. Gland Surg. 2020;9:S28-42.

58. Capdevila J, Wirth L, Ernst T, Ponce Aix S, Lin CC, Ramlau R, Butler MO, Delord JP, Gelderblom H, Ascierto PA, et al. PD-1 blockade in anaplastic thyroid carcinoma. J Clin Oncol. 2020:JCO1902727.

59. Coperchini F, Croce L, Marino M, Chiovato L, Rotondi M. Role of chemokine receptors in thyroid cancer and immunotherapy. Endocr Relat Cancer. 2019; 26:R465-78.

60. Meng X, Wang H, Zhao J, Hu L, Zhi J, Wei S, Ruan X, Hou X, Li D, Zhang J, et al. Apatinib inhibits cell proliferation and induces autophagy in human papillary thyroid carcinoma via the PI3K/Akt/mTOR signaling pathway. Front Oncol. 2020;10:217.

61. Yang J, Wang R, Li H, Lv Q, Meng W, Yang X. Lentivirus mediated RNA interference of EMMPRIN (CD147) gene inhibits the proliferation, matrigel invasion and tumor formation of breast cancer cells. Cancer Biomark. 2016; 17:237-47.

62. Yang XQ, Yang J, Wang R, Zhang S, Tan QW, Lv Q, Meng WT, Mo XM, Li HJ. Effect of specific silencing of EMMPRIN on the growth and cell cycle distribution of MCF-7 breast cancer cells. Genet Mol Res. 2015;14:15730-8.

63. Zhang RY, Wei D, Liu ZK, Yong YL, Wei W, Zhang ZY, LV JJ, Zhang Z, Chen $\mathrm{ZN}$, Bian $\mathrm{H}$. Doxycycline inducible chimeric antigen receptor $T$ cells targeting CD147 for hepatocellular carcinoma therapy. Front Cell Dev Biol. 2019;7:233.

64. Wang M, Zhang S, Sun Q, Yang X, Wang Y, Shang R, Zhu Y, Yao H, Li Y. Dual effects of an anti-CD147 antibody for esophageal cancer therapy. Cancer Biol Ther. 2019;20:1443-52.

65. Wang S, Liu C, Liu X, He Y, Shen D, Luo Q, Dong Y, Dong H, Pang Z. Effects of matrix metalloproteinase inhibitor doxycycline and CD147 antagonist peptide-9 on gallbladder carcinoma cell lines. Tumour Biol. 2017:39: 1010428317718192.

66. Fu ZG, Wang Y, Wang S, Shao D, Tian L, Li YX, Jiang JL, Chen ZN, Wen N. Synthesis and evaluation of a novel small-molecule compound as an anticancer inhibitor of CD147. Biomed Environ Sci. 2019;32:673-86.

67. Pahk K, Joung C, Song HY, Kim S, Kim WK. SP-8356, a novel inhibitor of CD147-cyclophilin a interactions, reduces plaque progression and stabilizes vulnerable plaques in apoE-deficient mice. Int J Mol Sci. 2019;21.

68. Pahk K, Noh H, Joung C, Jang M, Song HY, Kim KW, Han K, Hwang Jl, Kim S, Kim WK. A novel CD147 inhibitor, SP-8356, reduces neointimal hyperplasia and arterial stiffness in a rat model of partial carotid artery ligation. J Transl Med. 2019;17:274.

\section{Publisher's Note}

Springer Nature remains neutral with regard to jurisdictional claims in published maps and institutional affiliations.

Ready to submit your research? Choose BMC and benefit from:

- fast, convenient online submission

- thorough peer review by experienced researchers in your field

- rapid publication on acceptance

- support for research data, including large and complex data types

- gold Open Access which fosters wider collaboration and increased citations

- maximum visibility for your research: over $100 \mathrm{M}$ website views per year

At BMC, research is always in progress.

Learn more biomedcentral.com/submissions 